\title{
Revisiting the low-luminosity galaxy population of the NGC 5846 group with SDSS
}

\author{
P. Eigenthaler and W. W. Zeilinger
}

\author{
Institut für Astronomie, Universität Wien, Türkenschanzstraße 17, 1180 Vienna, Austria \\ e-mail: eigenthaler@astro.univie.ac.at
}

Received 22 September 2008 / Accepted 26 November 2009

\section{ABSTRACT}

\begin{abstract}
Context. Low-luminosity galaxies are known to outnumber the bright galaxy population in poor groups and clusters of galaxies. Yet, the investigation of low-luminosity galaxy populations outside the Local Group remains rare and the dependence on different group environments is still poorly understood. Previous investigations have uncovered the photometric scaling relations of early-type dwarfs and a strong dependence of morphology with environment.

Aims. The present study aims to analyse the photometric and spectroscopic properties of the low-luminosity galaxy population in the nearby, well-evolved and early-type dominated NGC 5846 group of galaxies. It is the third most massive aggregate of early-type galaxies after the Virgo and Fornax clusters in the local universe. Photometric scaling relations and the distribution of morphological types as well as the characteristics of emission-line galaxies are investigated.

Methods. Spectroscopically selected low-luminosity group members from the Sloan Digital Sky Survey with $c z<3000 \mathrm{~km} \mathrm{~s}^{-1}$ within a radius of $2^{\circ}=0.91 \mathrm{Mpc}$ around NGC 5846 are analysed. Surface brightness profiles of early-type galaxies are fit by a Sérsic model $\propto r^{1 / n}$. Star formation rates, oxygen abundances, and emission characteristics are determined for emission-line galaxies.

Results. Seven new group members showing no entry in previous catalogues are identified in the outer $\left(>1.33^{\circ}\right)$ parts of the system. Several photometric scaling relations for $\mathrm{dEs}$ as well as the morphology-density relation for dwarf galaxies are reproduced. The correlation between host and satellite morphologies in poor groups of galaxies is also confirmed. Nucleated dwarfs are found to be located in the vicinity to the brightest ellipticals in the group. Only two faint galaxies exhibit fine structure. Emission-line dwarfs show no interaction-induced activity.
\end{abstract}

Key words. galaxies: clusters: individual: NGC 5846 group - galaxies: dwarf - galaxies: photometry - methods: data analysis

\section{Introduction}

Redshift surveys indicate that most galaxies in the nearby universe are located in poor groups (Tully \& Fisher 1988). These aggregates not only consist of the ordinary bright Hubble types but also a low-luminosity dwarf galaxy population outnumbering their brighter counterparts by far. Previous observations studying the distribution and morphological properties of these low-luminosity galaxy populations have mainly focused on large-scale structures, e.g., the Virgo and Fornax clusters (Sandage \& Binggeli 1984; Ferguson 1989), which are representative of high-density environments. In contrast, poor groups serve as ideal laboratories for studying the environmental dependence of the properties of low-luminosity galaxy populations in a low-density environment where galaxy interactions, mergers, and the coalescence of individual galaxies are the primary physical processes. However, the acquisition of high quality photometry and spectroscopy of low-luminosity galaxies is challenging. Only the Local Group and a few other nearby galaxy aggregates have been investigated in detail to analyse the connection between low-luminosity galaxies and their environment (Karachentsev et al. 2002; Côté et al. 1997; Jerjen et al. 2000; Grützbauch et al. 2009). Because $\Lambda$ CDM cosmology proposes the hierarchical growth of galaxies by means of mergers, poor groups are commonly classified on the basis of their optical morphologies (Zabludoff 1999). Spiral dominated aggregates represent unevolved systems in this scenario, while aggregates containing tidal signatures and evidence of the formation of tidal dwarf galaxies mark an intermediate stage (Temporin et al. 2003). Groups dominated by early-type morphologies represent an advanced phase of coalescence. Dynamically, these wellevolved aggregates are more likely to be virialised systems than groups with a higher spiral fraction. The dwarf-to-giant ratio (DGR) is expected to increase with time because the bright, massive galaxies are more affected by mergers than their faint counterparts (Zabludoff \& Mulchaey 1998). However, the number of faint galaxies in groups and clusters is puzzling. The $\Lambda \mathrm{CDM}$ cosmology predicts more dwarfs than observed, an issue widely referred to as the missing satellite problem (Klypin et al. 1999).

This paper focuses on the low-luminosity galaxy population of the X-ray bright galaxy group around the giant elliptical NGC 5846 in the Local Supercluster. The group was first catalogued in de Vaucouleurs (1975). Since then, the system has appeared in many similar catalogues (Geller \& Huchra 1983; Garcia 1993). Zabludoff \& Mulchaey (1998) identified 13 dwarfs in the central region of the system. Mahdavi et al. (2005), hereafter [MTT05], identify $251 \pm 10$ group members including 83 spectroscopically confirmed ones. Carrasco et al. (2006) discovered 16 additional low surface brightness dwarfs. The NGC 5846 group of galaxies is located at a distance of $26.1 \mathrm{Mpc}$ in the Virgo III cloud of galaxies, a major component of the Local Supercluster (Tully 1982). Being an elongated structure extending from the Virgo cluster out from the Supergalactic Plane, this cloud contains about 40 luminous galaxies, the NGC 5846 system being the largest aggregate in the region. The NGC 5846 group is the most massive of only three dense groups 
(NGC 4274, NGC 5846, and M96) in the Local Supercluster that are dominated by elliptical and lenticular galaxies, and is the third most massive aggregate of early-type galaxies (after the Virgo and Fornax clusters) in the local universe. With an average galaxy density ${ }^{1}$ of $\rho=0.78 \pm 0.08 \mathrm{Mpc}^{-3}$ compared to $\rho=0.49 \pm 0.06 \mathrm{Mpc}^{-3}$ for the Local Group and $\rho \sim 5 \mathrm{Mpc}^{-3}$ for the central regions of the Virgo cluster, the NGC 5846 system also represents one of the densest galaxy environments found for poor groups. The group is dominated by early-type galaxies with a massive central elliptical surrounded by a symmetric X-ray halo. The large early-type fraction, the prevalence of dwarf galaxies together with the strong, and extended diffuse $\mathrm{X}$-ray emission indicate that the NGC 5846 group is an evolved system. The group consists of two smaller aggregates around the two brightest ellipticals NGC 5846 and NGC 5813. MTT05 determine the group virial mass to be $8.3 \pm 0.3 \times 10^{13} M_{\odot}$ by means of the median virial mass estimator proposed by Heisler et al. (1985) and derive a velocity dispersion of $322 \mathrm{~km} \mathrm{~s}^{-1}$.

The goals of this work are to study new faint members using the Sloan Digital Sky Survey (DR4) (Adelman-McCarthy et al. 2006) to extend the list of known group members and study photometric scaling relations, the distribution of morphological types, as well as the spectroscopic properties of the low-luminosity galaxy population. The paper is organized as follows. Section 2 provides a brief overview on the sample selection and data analysis procedures. Section 3 focuses on the low-luminosity galaxy population and presents the photometric and spectroscopic data of all individual objects. Finally, our results are summarized and discussed in Sect. 4. A group distance of 26.1 Mpc based on the work of MTT05 is used throughout this paper corresponding to a distance modulus of $m-M=32.08$. The systemic velocity of the group is assumed to be represented by that of NGC 5846, which is $v_{r}=1710 \mathrm{~km} \mathrm{~s}^{-1}$. To separate between dwarf and bright galaxies, an absolute magnitude of $M_{\mathrm{B}}=-16$ is used (Ferguson \& Binggeli 1994) except when otherwise stated. Total magnitudes presented in this work are SDSS model magnitudes ${ }^{2}$.

\section{Sample selection and data analysis}

To extend the existing sample of low-luminosity galaxies in the NGC 5846 system, we searched in the SDSS database for all galaxies with $c z<3000 \mathrm{~km} \mathrm{~s}^{-1}$ within a radius of $2^{\circ}=$ $0.91 \mathrm{Mpc}$, similar to the second turnaround radius $r_{2 t}=1.85^{\circ}$ presented by MTT05 ${ }^{3}$. Galaxies beyond these limits are very likely to be either field or background objects. The query resulted in a total sample of 74 galaxies, 19 bright objects and 55 dwarfs (see Table 1 and Fig. 1) with seven objects found in the outer parts $\left(>1.33^{\circ}\right)$ of the system that have no entry in previous catalogues. Though slightly fainter than $M_{\mathrm{B}}=-16$,

\footnotetext{
1 Densities taken from the Nearby Galaxies Catalog (Tully \& Fisher 1988), see Tully (1988) for details.

2 SDSS model magnitudes are based on a matched galaxy model that provides an optimal measure of the flux of a galaxy. The code fits a deVaucouleurs profile as well as an exponential profile to the two-dimensional image. The best-fit model is stored as the model magnitude.

3 Bound group members decouple from the Hubble flow at first turnaround, i.e., the zero-velocity surface around the group (Sandage 1986), begin to collapse, and again expand to a radius of second turnaround, finally oscillating and exchanging kinetic energy with other group members (Bertschinger 1985).
}

UGC 9751 and UGC 9760 are listed as bright members because of their classification as Scd and $\mathrm{Sd}_{\text {in }} \mathrm{NED}^{4}$.

Corrected FITS frames (bias subtracted, flat-fielded, and cleaned of bright stars) were extracted from the SDSS for these 55 dwarfs in the $g^{\prime}, r^{\prime}$, and $i^{\prime}$ bands ${ }^{5}$. SDSS model magnitudes were adopted from the database for each individual galaxy and transformed to the Johnson-Morgan-Cousins $B$ band by means of transformation equations from Smith et al. (2002). Absolute magnitudes were derived assuming an average extinction value of $A_{\mathrm{B}}=0.22$ from Schlegel et al. (1998) for the entire galaxy population. An isophote analysis was carried out for the dE subsample using the ellipse task within the IRAF stsdas package to fit ellipses to the galaxy images and measure the deviations from purely elliptical isophote shapes. A detailed description of the procedure is given by Jedrzejewski (1987). The analysis resulted in surface brightness profiles and the harmonic content of the isophotes. A Sérsic (1968) law $\mu(r)=\mu_{\mathrm{e}}+1.086 b_{n}\left(\left(r / r_{\mathrm{e}}\right)^{1 / n}-1\right)$ with an effective radius $r_{\mathrm{e}}$, an effective surface brightness $\mu_{\mathrm{e}}$, and a shape parameter $n$ as free parameters was fit to each object. The quantity $b_{n}$ is a function of $n$ and chosen so that half the galaxy luminosity is located within the effective radius ${ }^{6}$. The fitting was carried out using a Levenberg-Marquardt algorithm until a minimum in $\chi^{2}$ was achieved, yielding the parameters $n, r_{\mathrm{e}}$, and $\mu_{\mathrm{e}}$ with the corresponding uncertainties. The innermost parts of the surface brightness profiles $(\leq 1.4$ arcsec) have not been taken into account for the fitting procedure to avoid the effects of seeing. Central surface brightnesses $\mu_{0_{B}}$ were derived from the fit. The results of the isophote analysis are presented in Table 2. Sérsic models were subtracted from the original images yielding residual frames that were investigated for photometric substructures. In addition to the photometric data, wavelength and flux-calibrated spectra (sky subtracted and corrected for telluric absorption) were examined for all sample galaxies. The spectra cover a wavelength range of $\lambda \lambda 3800-9200 \AA$ and were checked for spectral lines in both emission and absorption. Identified spectral lines were fit by Gaussians yielding central wavelengths and full widths at half maximum (FWHM). The derived central wavelengths were subsequently used to verify SDSS redshifts.

\section{The low-luminosity galaxy population}

Table 1 lists the NGC 5846 galaxy group sample studied in this work. Morphological types for dwarfs were classified by the visual inspection of SDSS images and spectra. Objects with a smooth light distribution and exponential profiles were classified as dEs. The dE ellipticity was determined from the isophote analysis. Dwarfs showing a blue patchy appearance and emission lines were classified as dIrrs. Morphological types for bright galaxies were taken from NED. Dwarfs are identified with increasing projected radial distance from NGC 5846.

We extend previous group member lists with seven new objects in the outer parts $\left(>1.33^{\circ}\right)$ of the system that have no entry in previous catalogues. Despite exhibiting slightly brighter values than $M_{\mathrm{B}}=-16$, some galaxies were classified as dwarfs because of their nearly exponential surface brightness profiles

\footnotetext{
4 http://nedwww.ipac. caltech.edu/

5 See Fukugita et al. (1996) for a description of the SDSS photometric system.

6 The exact relation between $n$ and $b_{n}$ is derived by $\Gamma(2 n)=\gamma\left(2 n, b_{n}\right)$, where $\Gamma(a)$ and $\gamma(a, x)$ are the complete and incomplete gamma functions, respectively. A good approximation is $b_{n}=1.9908 n-0.3118$ for $0.5 \leq n \leq 2$, which has been used in this work.
} 
Table 1. Galaxies within $2^{\circ}$ around NGC 5846 as studied in this work.

\begin{tabular}{|c|c|c|c|c|c|c|c|c|c|}
\hline Galaxy & {$[\mathrm{MTT} 05]^{a}$} & $\alpha_{2000^{b}}$ & $\delta_{2000^{b}}$ & $d[\operatorname{arcmin}]^{c}$ & $c z\left[\mathrm{~km} \mathrm{~s}^{-1}\right]$ & $i^{\prime b}$ & $r^{\prime}-i^{\prime b}$ & $M_{\mathrm{B}}$ & Type \\
\hline CGCG 20-39 & 021 & 145848.71 & 020124.6 & 117.8 & $1800 \pm 30$ & 13.25 & 0.39 & $\begin{array}{l}-17.39 \\
\end{array}$ & $E$ \\
\hline NGC 5806 & 037 & 150000.39 & 015328.7 & 98.7 & $1350 \pm 30$ & 11.41 & 0.46 & -19.03 & $\mathrm{SAB}(\mathrm{s}) \mathrm{b}$ \\
\hline N5846_55 & 042 & 150016.58 & 021802.6 & 102.1 & $1830 \pm 30$ & 14.19 & 0.36 & -16.55 & S0 \\
\hline NGC 5811 & 046 & 150027.40 & 013724.1 & 90.5 & $1529 \pm 15$ & 13.83 & 0.31 & -17.30 & $\mathrm{SB}(\mathrm{s}) \mathrm{m}$ \\
\hline N5846_53 & 048 & 150033.03 & 021349.2 & 96.6 & $1260 \pm 30$ & 16.03 & 0.27 & -14.83 & $\mathrm{dE}, \mathrm{N}$ \\
\hline N5846_47 & 055 & 150052.59 & 012417.7 & 85.0 & $1890 \pm 60$ & 15.35 & 0.35 & -15.37 & $\mathrm{dE} 5, \mathrm{~N}$ \\
\hline N5846_45 & 058 & 150059.36 & 015236.2 & 84.1 & $2190 \pm 90$ & 17.56 & 0.28 & -13.32 & $\mathrm{dE}, \mathrm{N}$ \\
\hline N5846_43 & 059 & 150059.36 & 013857.1 & 82.5 & $2430 \pm 60$ & 16.80 & 0.41 & -13.84 & $\mathrm{dE} 1$ \\
\hline N5846_49 & 060 & 150100.86 & 010049.8 & 89.5 & $1740 \pm 30$ & 17.48 & -0.10 & -14.15 & dIrr \\
\hline N5846_54 & 061 & 150103.11 & 004227.4 & 97.7 & $1770 \pm 60$ & 14.69 & 0.32 & -16.20 & S0/a \\
\hline N5846_48 & 063 & 150106.96 & 020525.2 & 85.7 & $1950 \pm 60$ & 17.11 & 0.24 & -13.74 & $\mathrm{dE}$ \\
\hline NGC 5813 & 064 & 150111.23 & 014207.1 & 79.7 & $1973 \pm 06$ & 10.75 & 0.46 & -19.63 & E1-2 \\
\hline N5846_39 & 068 & 150115.33 & 012953.5 & 78.8 & $2220 \pm 90$ & 16.57 & 0.27 & -14.62 & $\mathrm{dIrr}$ \\
\hline N5846_40 & 069 & 150115.89 & 014624.5 & 79.0 & $1500 \pm 60$ & 16.73 & 0.27 & -14.24 & $\mathrm{dE} 5$ \\
\hline N5846_37 & 073 & 150138.38 & 014319.8 & 73.1 & $2280 \pm 60$ & 16.47 & 0.40 & -14.22 & $\mathrm{dE} 2, \mathrm{~N}$ \\
\hline N5846_38 & 075 & 150138.61 & 015212.6 & 74.4 & $2160 \pm 30$ & 16.42 & 0.27 & -14.62 & dIrr \\
\hline UGC 9661 & 083 & 150203.50 & 015028.6 & 67.9 & $1241 \pm 06$ & 13.62 & 0.24 & -17.55 & $\mathrm{SB}(\mathrm{rs}) \mathrm{d}$ \\
\hline N5846_29 & 088 & 150228.13 & 012151.1 & 62.0 & $1470 \pm 90$ & 17.11 & 0.25 & -13.95 & $\mathrm{dE}$ \\
\hline N5846_30 & 090 & 150233.02 & 015608.2 & 62.3 & $1650 \pm 60$ & 17.07 & 0.24 & -13.93 & $\mathrm{dE}$ \\
\hline N5846_31 & 091 & 150236.03 & 020139.5 & 63.6 & $1980 \pm 60$ & 16.87 & 0.33 & -13.99 & dE5 \\
\hline N5846_35 & 113 & 150344.28 & 023308.1 & 70.2 & $1770 \pm 60$ & 16.21 & 0.29 & -14.65 & $\mathrm{dE} 1$ \\
\hline N5846_26 & 114 & 150349.93 & 005831.7 & 54.9 & $2010 \pm 90$ & 15.88 & 0.07 & -15.65 & dIrr \\
\hline N5846_25 & 115 & 150350.31 & 010736.5 & 49.0 & $1590 \pm 30$ & 14.85 & 0.39 & -15.79 & $\mathrm{dE} 3$ \\
\hline N5846_41/42 & - & 150355.94 & 002551.1 & 80.2 & $1600 \pm 300$ & 15.80 & -0.09 & -16.23 & dIrr \\
\hline NGC 5831 & 122 & 150406.99 & 011311.7 & 42.4 & $1655 \pm 03$ & 11.20 & 0.45 & -19.26 & E3 \\
\hline N5846_20 & 124 & 150408.45 & 013128.0 & 35.5 & $1860 \pm 30$ & 15.15 & 0.26 & -15.99 & dIrr \\
\hline N5846_32 & 125 & 150413.07 & 023234.6 & 65.7 & $1890 \pm 60$ & 14.48 & 0.39 & -16.22 & $\mathrm{dE} 2$ \\
\hline N5846_23 & 132 & 150424.69 & 020652.5 & 43.6 & $1770 \pm 30$ & 16.05 & 0.32 & -14.81 & $\mathrm{dE} 4$ \\
\hline N5846_16 & 142 & 150442.90 & 011727.2 & 32.6 & $1980 \pm 60$ & 15.39 & 0.40 & -15.31 & $\mathrm{dE} 1$ \\
\hline N5846_18 & 144 & 150448.50 & 015850.6 & 33.8 & $1950 \pm 30$ & 17.09 & 0.19 & -14.07 & dIrr \\
\hline N5846_14 & 148 & 150504.40 & 015751.5 & 30.2 & $2368 \pm 05$ & 16.54 & 0.14 & -14.82 & dIrr \\
\hline NGC 5838 & 159 & 150526.23 & 020557.4 & 33.6 & $1358 \pm 09$ & 10.67 & 0.49 & -19.69 & SA0 \\
\hline NGC 5839 & 160 & 150527.48 & 013805.3 & 15.5 & $1226 \pm 15$ & 11.90 & 0.44 & -18.57 & SAB(rs) \\
\hline N5846_06 & 162 & 150528.74 & 011733.1 & 24.1 & $2308 \pm 05$ & 17.65 & -0.03 & -14.04 & dIrr \\
\hline N5846_02 & 165 & 150531.83 & 013515.4 & 14.4 & $0900 \pm 30$ & 16.63 & 0.30 & -14.23 & $\mathrm{dE} 2, \mathrm{~N}$ \\
\hline N5846_05 & 167 & 150537.73 & 011811.2 & 22.3 & $2040 \pm 60$ & 16.02 & 0.34 & -14.82 & $\mathrm{dE} 1$ \\
\hline N5846_04 & 177 & 150550.57 & 015429.7 & 20.6 & $1770 \pm 30$ & 15.52 & 0.35 & -15.19 & $\mathrm{dE} 1$ \\
\hline N5846_09 & 180 & 150553.25 & 020027.0 & 25.7 & $1290 \pm 30$ & 17.33 & 0.12 & -14.15 & dIrr \\
\hline NGC 5845 & 184 & 150600.78 & 013801.6 & 7.3 & $1451 \pm 09$ & 11.78 & 0.47 & -18.68 & $\mathrm{E}$ \\
\hline N5846_19 & 187 & 150603.32 & 021105.5 & 35.4 & $1620 \pm 60$ & 15.44 & 0.02 & -16.00 & dIrr \\
\hline N5846_03 & 191 & 150606.73 & 011920.8 & 17.9 & $2340 \pm 60$ & 16.63 & 0.32 & -14.18 & $\mathrm{dE} 4$ \\
\hline N5846_12 & 192 & 150611.32 & 020546.4 & 29.8 & $1799 \pm 05$ & 16.01 & 0.05 & -15.72 & dIrr \\
\hline NGC 5846 A & 201 & 150629.19 & 013541.5 & 0.6 & $2200 \pm 15$ & 13.56 & 0.41 & -17.02 & $\mathrm{cE} 2-3$ \\
\hline NGC 5846 & 202 & 150629.28 & 013620.2 & 0.0 & $1710 \pm 60$ & 10.43 & 0.48 & -19.96 & E0-1 \\
\hline N5846_44 & - & 150634.25 & 001255.7 & 83.4 & $2010 \pm 90$ & 16.76 & 0.28 & -14.26 & $\mathrm{dE}, \mathrm{N}$ \\
\hline N5846_01 & 205 & 150634.27 & 013331.6 & 03.1 & $1500 \pm 30$ & 14.56 & 0.45 & -15.90 & $\mathrm{dE} 1$ \\
\hline NGC 5848 & 206 & 150635.03 & 020017.3 & 24.0 & $1260 \pm 30$ & 12.96 & 0.40 & -17.67 & S0 \\
\hline N5846_50 & - & 150640.97 & 000436.3 & 91.8 & $1710 \pm 30$ & 16.79 & 0.28 & -14.11 & $\mathrm{dE} 3$ \\
\hline N5846_27 & 212 & 150642.03 & 003803.7 & 58.4 & $2340 \pm 60$ & 17.00 & 0.23 & -14.26 & dIrr \\
\hline NGC 5850 & 233 & 150707.68 & 013239.2 & 10.3 & $2550 \pm 60$ & 11.46 & 0.49 & -18.87 & $\mathrm{SB}(\mathrm{r}) \mathrm{b}$ \\
\hline N5846_17 & 241 & 150734.18 & 010713.1 & 33.3 & $1620 \pm 30$ & 16.52 & 0.26 & -14.54 & $\mathrm{dE} 4$ \\
\hline N5846_13 & 244 & 150737.23 & 020109.4 & 30.1 & $1890 \pm 60$ & 14.61 & 0.38 & -16.13 & $\mathrm{dE} 1, \mathrm{~N}$ \\
\hline NGC 5854 & 246 & 150747.69 & 023407.0 & 61.0 & $1736 \pm 09$ & 11.64 & 0.39 & -19.09 & $\mathrm{SB}(\mathrm{s}) 0$ \\
\hline N5846_11 & 247 & 150747.82 & 011731.4 & 27.2 & $2100 \pm 60$ & 14.61 & 0.35 & -16.22 & $\mathrm{dE} 2, \mathrm{~N}$ \\
\hline N5846_22 & 252 & 150801.37 & 020903.8 & 40.0 & $1080 \pm 30$ & 16.57 & 0.35 & -14.23 & $\mathrm{dE} 2$ \\
\hline N5846_07 & 256 & 150805.61 & 013905.7 & 24.2 & $1830 \pm 30$ & 14.40 & 0.38 & -16.16 & S0/a \\
\hline N5846_08 & 260 & 150809.25 & 013629.7 & 25.0 & $2130 \pm 60$ & 15.31 & 0.32 & -15.53 & dIrr \\
\hline N5846_10 & 261 & 150812.38 & 012958.8 & 26.5 & $1620 \pm 30$ & 16.69 & 0.36 & -14.10 & $\mathrm{dE} 1, \mathrm{~N}$ \\
\hline N5846_15 & 266 & 150822.69 & 014754.9 & 30.6 & $1680 \pm 60$ & 16.72 & 0.27 & -14.20 & $\mathrm{dE} 4, \mathrm{~N}$ \\
\hline N5846_21 & 276 & 150847.18 & 015359.8 & 38.7 & $2010 \pm 60$ & 15.37 & 0.32 & -15.50 & dE6 \\
\hline N5846_28 & 283 & 150904.29 & 004919.1 & 60.9 & $1649 \pm 05$ & 15.60 & 0.30 & -15.34 & dIrr \\
\hline N5846_33 & 287 & 150907.86 & 004329.5 & 66.1 & $1680 \pm 30$ & 16.64 & 0.24 & -14.43 & dIrr \\
\hline N5846_24 & 290 & 150914.97 & 015517.1 & 45.5 & $1740 \pm 30$ & 15.40 & 0.31 & -15.44 & $\mathrm{dE} 4$ \\
\hline NGC 5864 & 299 & 150933.56 & 030309.9 & 98.3 & $1800 \pm 30$ & 11.42 & 0.40 & -19.21 & $\mathrm{SB}(\mathrm{s}) \mathrm{sp}$ \\
\hline NGC 5869 & - & 150949.41 & 002812.2 & 84.5 & $1934 \pm 15$ & 11.48 & 0.39 & -19.01 & So \\
\hline UGC 9746 & 305 & 151016.54 & 015603.3 & 60.1 & $1830 \pm 60$ & 13.64 & 0.33 & -17.24 & Sbc \\
\hline
\end{tabular}


Table 1. continued.

\begin{tabular}{cccccccccc}
\hline \hline Galaxy & ${\text { [MTT05 }]^{a}}^{2} \alpha_{2000^{b}}$ & $\delta_{2000}{ }^{b}$ & $d$ [arcmin $^{c}$ & $c z\left[\mathrm{~km} \mathrm{~s}^{-1}\right]$ & $i^{\prime b}$ & $r^{\prime}-i^{\prime b}$ & $M_{\mathrm{B}}$ & Type \\
\hline UGC 9751 & 311 & 151058.44 & 012615.9 & 68.0 & $1574 \pm 15$ & 15.31 & 0.26 & -15.80 & Scd \\
N5846_34 & 313 & 151101.33 & 014050.1 & 68.2 & $1710 \pm 30$ & 16.03 & 0.32 & -14.81 & dE2 \\
N5846_36 & 317 & 151121.63 & 013637.6 & 73.1 & $1950 \pm 90$ & 16.27 & 0.21 & -14.70 & dE \\
UGC 9760 & 321 & 151202.18 & 014151.3 & 83.4 & $2024 \pm 03$ & 14.85 & 0.38 & -16.15 & Sd \\
N5846_46 & 323 & 151208.15 & 013508.6 & 84.7 & $2010 \pm 60$ & 15.85 & 0.28 & -15.25 & dE1 \\
N5846_52 & - & 151224.05 & 020448.2 & 93.1 & $1740 \pm 30$ & 15.69 & 0.32 & -15.17 & dE3,N \\
N5846_56 & - & 151231.74 & 004845.3 & 102.3 & $1830 \pm 60$ & 15.14 & 0.32 & -15.82 & dIrr \\
N5846_51 & - & 151241.39 & 013723.7 & 93.0 & $1920 \pm 60$ & 16.16 & 0.28 & -14.80 & dE4 \\
\hline
\end{tabular}

Notes. ${ }^{(a)}$ Galaxy identification from Mahdavi et al. (2005); ${ }^{(b)}$ coordinates and magnitudes taken from SDSS DR4; ${ }^{(c)}$ projected radial distance to NGC 5846; ${ }^{(d)}$ N5846_41/42 are two HII regions classified as individual galaxies in SDSS (see Sect. 3.2).

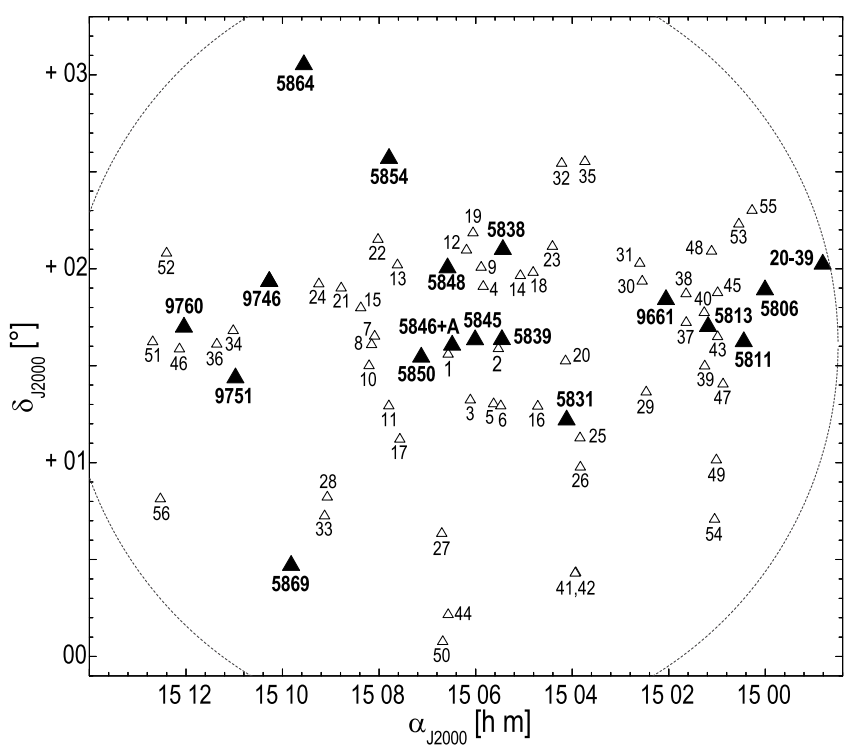

Fig. 1. Projected spatial distribution of NGC 5846 group members as studied in this work. Filled triangles indicate bright members, while open ones represent the low-luminosity galaxies. Numbers refer to the galaxy identification of Table 1 . The circle shows the $2^{\circ}(0.91 \mathrm{Mpc})$ query radius.

or their compactness. Thirty-five dwarfs $(\sim 63 \%)$ were identified as dEs according to their optical appearance and red colours $(B-V)_{0} \simeq 0.85$. Eighteen objects $(\sim 32 \%)$ are dwarf irregulars with a patchy, blue optical appearance and emission lines, both indicative of ongoing star formation. Three galaxies $(\sim 1 \%)$ exhibit photometric fine structure and sizes that are in-between those of dwarf and bright galaxies. Eleven dwarfs ( $\sim 31 \%$ of the $\mathrm{dEs}$ ) are found to be nucleated on the basis of the analysis of their surface brightness profiles.

Our list of nucleated dwarfs is almost identical to the list obtained by MTT05 with exception of only three objects classified differently. Taking the Schechter luminosity function derived by MTT05, we conclude that with an absolute magnitude of $M_{\mathrm{B}}=-13.32$ for our faintest object, our sample consists of at most $34 \%$ of the total number of galaxies expected in the system to a limiting magnitude of $M_{\mathrm{B}}=-10$. Our sample yields a dwarf-to-giant-ratio (DGR) of $\sim 3$ as lower limit. Following the photometric dwarf classification of MTT05, this value could rise to $\sim 12$, confirming that the NGC 5846 group is indeed one of the richest groups in the Local Supercluster.

The projected spatial distribution of all investigated galaxies is illustrated in Fig. 1. The distribution indicates two substructures of the system around the two brightest ellipticals

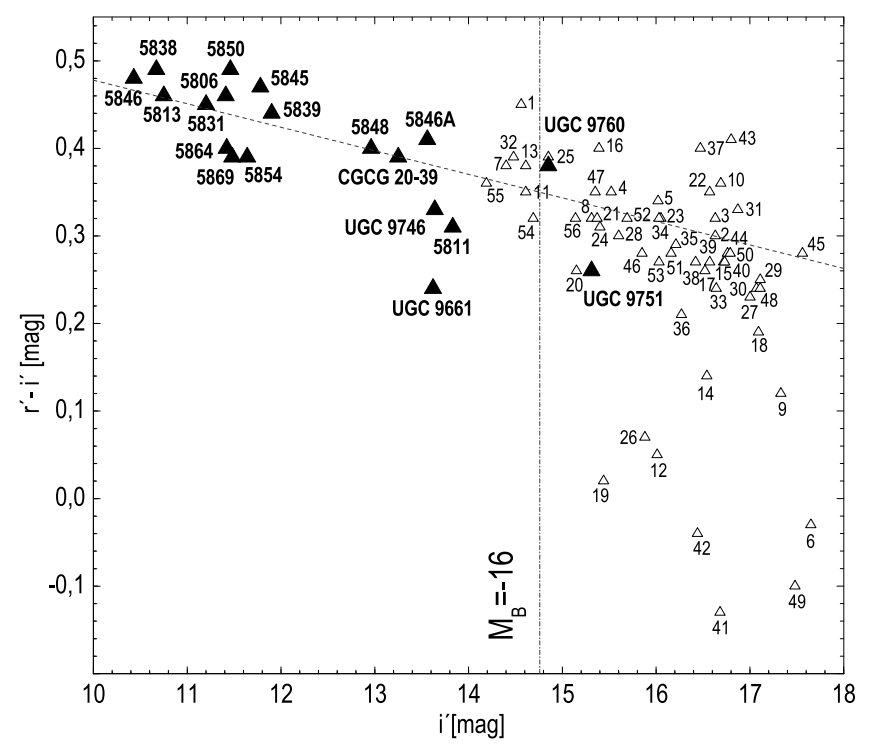

Fig. 2. Colour-magnitude diagram of NGC 5846 group members. Numbers as in Fig. 1. The dashed line indicates the redsequence linear fit to bright galaxies and early-type dwarfs: $r^{\prime}-i^{\prime}=$ $0.75( \pm 0.05)-0.027( \pm 0.003)$. The vertical line separates dwarfs from bright galaxies.

NGC 5846 and NGC 5813. A third, much smaller subgroup can be discerned around UGC 9760, which in contrast to the NGC 5846 and NGC 5813 aggregates, has no noteworthy X-ray counterpart. We present in Fig. 2 a colour-magnitude diagram for the studied group galaxy population. The diagram indicates the prevalence of an early-type dwarf morphology and the continuation of a red sequence similar to that found in galaxy clusters (Gladders et al. 1998) into the dwarf regime. Figure 3 shows the $n-M_{\mathrm{B}}$ relation consisting of our $\mathrm{dE}$ sample as well as the bright galaxies classified as ellipticals in NED. Sérsic shape parameters of the low-luminosity galaxy population show a mean value of $n=1.19$ with a scatter of $\sigma_{n}=0.23$ representing nearly exponential surface brightness profiles as expected for dwarf ellipticals. Three dwarfs (N5846_10, N5846_15, N5846_43) show comparatively large error bars of the shape parameter because of their faintness or contamination of their surface brightness profiles by a central nucleus. Our data confirm the trend of more luminous galaxies having higher shape parameters, thus a steeper surface brightness profile than the less luminous objects. Interestingly there are no objects with intermediate Sérsic parameters between $2<n<3$. NGC 5813 shows a comparatively high shape parameter around $n \sim 8$. 


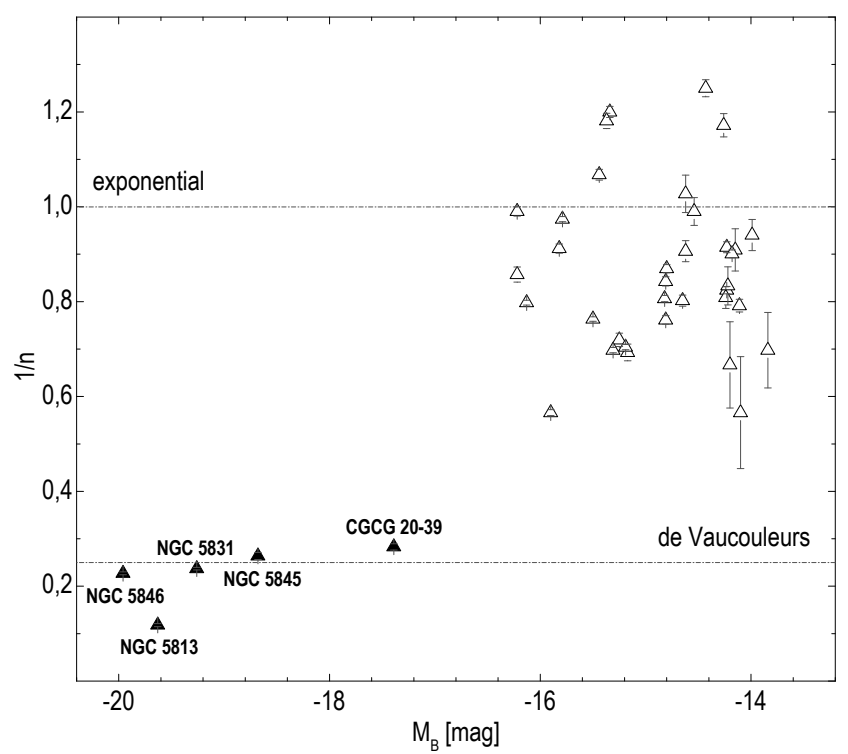

Fig. 3. Sérsic shape parameter versus absolute blue magnitude of earlytype galaxies in the NGC 5846 group. Open symbols represent dwarf ellipticals, while filled triangles show bright ellipticals. The horizontal lines indicate exponential and de Vaucouleurs $r^{1 / 4}$ laws.

\subsection{Scaling relations}

When addressing the question of the influence of the environment on the evolution of galaxies, scaling relations of the photometric properties of early-type galaxies are a useful tool. However, the environment does not seem to affect all early-type systems in a similar way. The fundamental plane of bright ellipticals is found to be almost independent of environment, while dwarf ellipticals exhibit a stronger environmental dependence (Nieto et al. 1990; Peterson \& Caldwell 1993). Elliptical galaxies and bulges are known to exhibit a tight correlation in the $\mu-M_{\mathrm{B}}$ plane such that higher surface brightnesses correspond to lower total luminosities (Kormendy 1985; Kormendy et al. 2009). Dwarf galaxies exhibit an opposite trend, being a distinct class of objects with possibly different formation and evolution histories. Binggeli \& Jerjen (1998), hereafter [BJ98] investigated the $\mu-B$ plane for Virgo cluster early-type dwarfs and confirm this trend. Figure 4(a) shows the $\mu_{0_{B}}-M_{\mathrm{B}}$ plane for our dEs and the relationship determined by BJ98 (dash-dotted line) for their sample of Virgo dwarf ellipticals (assuming a Virgo distance of 16.5 Mpc corresponding to a distance modulus of $~ 31.09$ ). The figures Figs. 4(b)-(f) focus on $M_{\mathrm{B}}, \log n, \mu_{0_{B}}, \log r_{0}$, and ellipticity $\epsilon$. Sérsic shape parameters used here should not be compared directly with those of BJ98, because $n=1 / n_{\mathrm{BJ} 98}$. The parameter $r_{0}$ is the scale radius of the Sérsic model $I(r)=I_{0} \exp \left[-r / r_{0}\right]^{1 / n}$. Table 2 presents the corresponding values of the isophote analysis. Objects with larger errors than the data itself are not listed.

Our data in the $\mu_{0_{B}}-M_{\mathrm{B}}$ plane are in agreement with the work of BJ98. One object falling outside the $2 \sigma$ limit is N5846_01, which has a comparatively too high surface brightness according to its luminosity (see Sect. 3.2). In the $\log n-M_{\mathrm{B}}$ plane, our data show a larger scatter $\sigma_{\log n}=0.19$. Compared to the dwarf sample of the Virgo cluster, our dwarfs agree with the Virgo dwarf relation, however. Our sample also confirms a trend between shape parameter $n$ and scale radius $r_{0}$ with smaller objects exhibiting higher shape parameters. Again N5846_01 is the only object in our sample to fall away from the trend. The scale radius $r_{0}$ is also strongly correlated with the central surface brightness as seen in the $\mu_{0_{B}}-\log r_{0}$ plane. The NGC 5846 dwarfs follow the relation obtained for the Virgo dwarfs with a lower scatter in magnitude $\left(\sigma=0.83 \mathrm{mag} \operatorname{arcsec}^{-2}\right)$ than that measured by BJ98, who derive a value of $\sigma=1.25 \mathrm{mag} \operatorname{arcsec}^{-2}$. As for BJ98, we also derived the best-fit linear combination between shape parameter $n$, central surface brightness $\mu_{0}$, and absolute $B$ band magnitude with a scatter of $\sigma=0.59 \mathrm{mag}$. Finally, we also checked whether a correlation exists between shape parameter $n$ and ellipticity. There is a slight trend of flattened objects showing shallower surface brightness profiles.

The structural properties of early-type systems can in addition be investigated by the Hamabe \& Kormendy (1987) relation, a photometric projection of the fundamental plane of galaxies relating the logarithm of effective radii and effective surface brightnesses linearly (Ziegler et al. 1999; di Serego Alighieri et al. 2005). This relation divides early-type systems into regular and bright classes separated at the effective radius of $r_{\mathrm{e}} \simeq 3 \mathrm{kpc}$ (Capaccioli et al. 1992). In hierarchical evolution scenarios, regular galaxies are understood to be the progenitors of bright ones that evolve through successive mergers along the HamabeKormendy relation (Capelato et al. 1995). However, the numerical simulations of Evstigneeva et al. (2004) suggest that low mass systems such as dwarf galaxies can only follow this scenario when a large amount of dissipation is involved. Figure 5 shows the Hamabe-Kormendy relation for the faint galaxy population of our work. Open squares are dwarfs from X-ray faint and X-ray bright groups studied by Khosroshahi et al. (2004). All faint galaxies of our sample are regular systems with similar properties to those of the dwarfs from Khosroshahi et al. (2004): this suggests that there is no strong difference in structural properties of the early-type dwarfs between the NGC 5846 group and other group environments. Again, N5846_01 is the only dwarf clearly separated from the main cloud of dEs.

\subsection{Comments on individual objects}

Most of the dwarfs investigated do not have any photometric or spectroscopic peculiarities, although a few galaxies in the sample deserve a closer examination. Figure 6 presents all objects that clearly exhibit fine structure in their optical appearance with $r^{\prime}$ band images and residual frames shown for each galaxy. Because of their relatively large angular diameters, the sizes of these objects are in-between those of dwarf and bright galaxies.

N5846_01 is the closest dwarf elliptical to NGC 5846 with a much higher central surface brightness than the remainder of our dwarfs. Furthermore it is the reddest galaxy in our low-luminosity galaxy sample. With an average effective radius of only $r_{\mathrm{e}} \sim 200 \mathrm{pc}$ in all studied passbands, this object is also the most compact of our sample. Because of its visual appearance and close proximity to NGC 5846, MTT05 suggested that N5846_01 has probably been affected by tidal stripping and should therefore be classified instead as an ultra compact dwarf. This idea is also supported by the nearby object NGC 5846A exhibiting a similar morphological appearance. Indeed, NGC 5846A is an extremely rare compact elliptical similar to M 32, which is the prototypical object of this class. Thus, N5846_01 is perfectly consistent with a scenario in which the structural differences of compact ellipticals and ordinary dEs are produced when the compacts form within the potential well of another massive galaxy, whereas dEs evolve as isolated systems (Burkert 1994).

N5846_07 is classified as an S0/a galaxy by MTT05 and was originally considered as an almost certainly background object in their sample because of its optical appearance. Spectroscopy 
A\&A 511, A12 (2010)
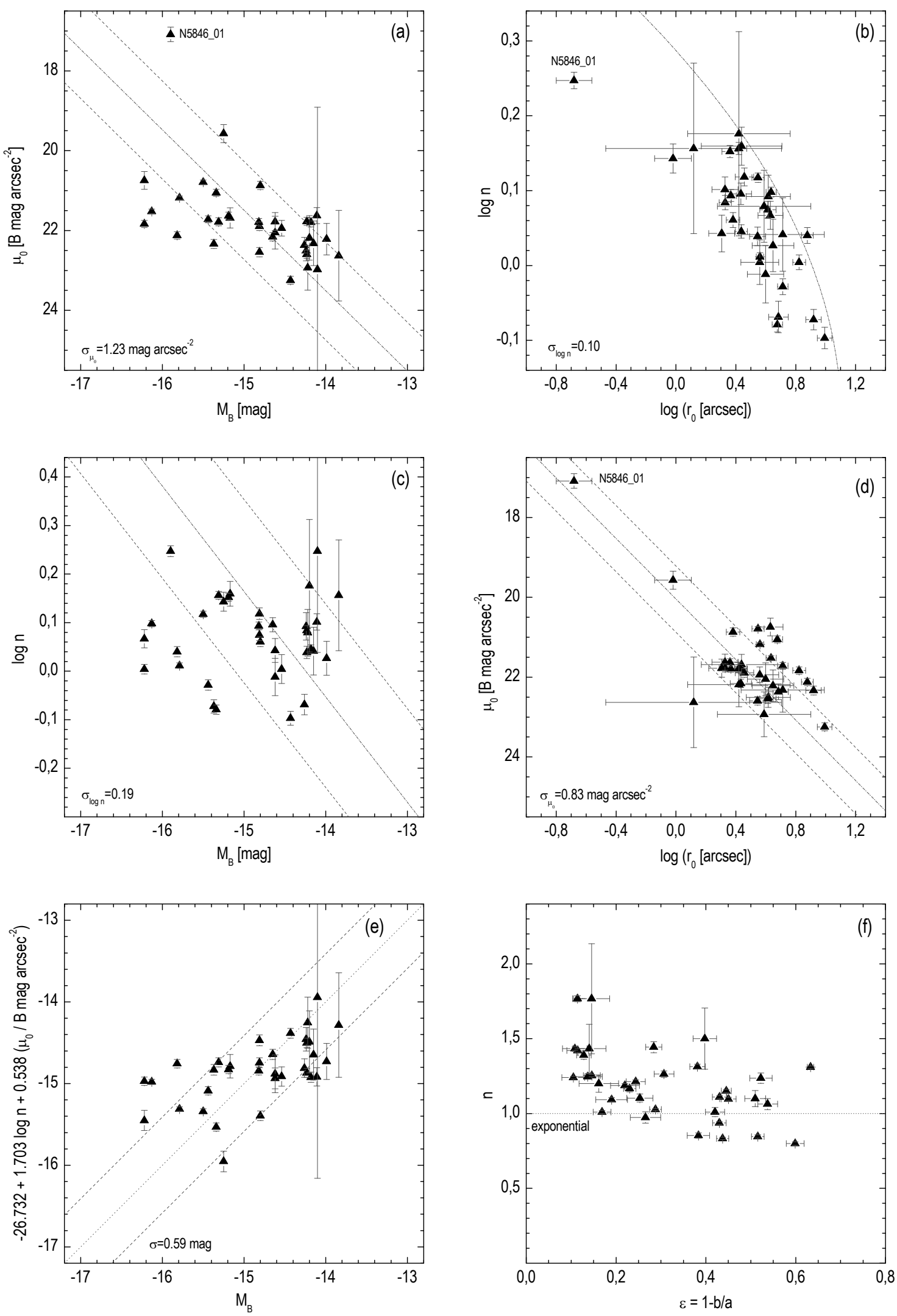

Fig. 4. Photometric scaling relations of the low-luminosity galaxy population in the NGC 5846 group. In panels a)-d), dash-dotted lines are the relations of the Virgo cluster early-type dwarfs from BJ98 assuming a distance to the Virgo cluster of $16.5 \mathrm{Mpc}$. The scatter in our data with respect to the BJ98 relations is given in the lower left corner of each panel. One- $\sigma$ deviations are indicated as dashed lines. Panel a): $\mu_{0_{B}}-M_{\mathrm{B}}$ plane. N5846_01, classified as ultra compact dwarf by MTT05 is located outside $2 \sigma$. Panel e): best-fit linear combination of shape parameter $n$ and central surface brightness $\mu_{0}$ with respect to absolute magnitude $M_{\mathrm{B}}$. N5846_01 was excluded from the fit and is not shown in the diagram. The dotted line indicates the identity. f): Sérsic shape parameter versus ellipticity. Objects with high ellipticity tend to have shallower surface brightness profiles. The dotted line indicates an exponential profile. 
Table 2. Surface photometry of the low-luminosity galaxy sample.

\begin{tabular}{|c|c|c|c|c|c|c|c|c|c|c|}
\hline Galaxy & $n_{i^{\prime}}$ & $n_{r^{\prime}}$ & $n_{g^{\prime}}$ & $\mu_{0_{B}}$ & $r_{e_{i^{\prime}}}$ & $r_{e_{r^{\prime}}}$ & $r_{e_{g^{\prime}}}$ & $\chi_{v, i^{\prime}}^{2}$ & $\chi_{v, r^{\prime}}^{2}$ & $\chi_{v, g^{\prime}}^{2}$ \\
\hline N5846_01 & $1.84(4)$ & $1.82(3)$ & $1.64(3)$ & $17.1(2)$ & $1.6(4)$ & $1.7(3)$ & $1.6(3)$ & 0.00043 & 0.00025 & 0.00055 \\
\hline N5846_02 & $1.12(3)$ & $1.06(2)$ & $1.10(2)$ & $22.6(1)$ & $6.9(8)$ & $6.9(6)$ & $7.0(5)$ & 0.00528 & 0.00317 & 0.00229 \\
\hline N5846_03 & $1.08(1)$ & $1.14(2)$ & $1.11(2)$ & $21.8(1)$ & $5.1(2)$ & $5.7(4)$ & $5.9(5)$ & 0.0018 & 0.00325 & 0.00221 \\
\hline N5846_04 & $1.43(2)$ & $1.53(2)$ & $1.30(2)$ & 21.63(9) & $8.4(9)$ & $9.5(9)$ & $7.6(5)$ & 0.00159 & 0.00064 & 0.00099 \\
\hline N5846_05 & $1.31(2)$ & $1.27(1)$ & $1.14(2)$ & 21.79(9) & $6.1(5)$ & $6.3(3)$ & $5.7(3)$ & 0.00288 & 0.00109 & 0.00217 \\
\hline N5846_09 & $0.91(9)$ & $1.09(9)$ & $1.3(1)$ & $22.3(6)$ & $9(3)$ & $10(4)$ & $12(7)$ & 0.00681 & 0.00957 & 0.0078 \\
\hline N5846_10 & $1.4(3)$ & $2.0(7)$ & $1.9(8)$ & $23(4)$ & - & - & - & 0.00468 & 0.00335 & 0.00513 \\
\hline N5846_11 & $1.01(3)$ & $1.15(3)$ & $1.34(5)$ & $20.7(2)$ & $9.0(9)$ & $10(1)$ & $10(2)$ & 0.00824 & 0.00925 & 0.01286 \\
\hline N5846_13 & $1.22(2)$ & $1.33(1)$ & $1.21(1)$ & $21.52(6)$ & $11.3(8)$ & $12.3(5)$ & $10.9(7)$ & 0.00286 & 0.00065 & 0.00066 \\
\hline N5846_15 & $1.8(6)$ & $1.20(8)$ & $1.5(1)$ & $22.2(6)$ & - & $10(3)$ & $13(7)$ & 0.0038 & 0.00293 & 0.00142 \\
\hline N5846_16 & $1.42(2)$ & $1.45(2)$ & $1.43(2)$ & 21.8(1) & $10.0(9)$ & $10.1(8)$ & $10(1)$ & 0.001 & 0.00069 & 0.00106 \\
\hline N5846_17 & $0.97(6)$ & $1.11(6)$ & $0.95(3)$ & $21.9(2)$ & $6(1)$ & $7(2)$ & $5.6(7)$ & 0.00375 & 0.00238 & 0.00143 \\
\hline N5846_21 & $1.37(2)$ & $1.21(1)$ & $1.35(1)$ & $20.79(6)$ & 11(1) & $9.7(5)$ & $10.8(6)$ & 0.00332 & 0.00085 & 0.0015 \\
\hline N5846_22 & $1.23(2)$ & $1.23(2)$ & $1.18(2)$ & $21.8(1)$ & $5.2(4)$ & $5.3(3)$ & $5.3(4)$ & 0.00354 & 0.00212 & 0.00356 \\
\hline N5846_23 & $1.41(3)$ & $1.31(3)$ & $1.22(2)$ & $21.9(1)$ & $9(1)$ & $8(1)$ & $8.7(7)$ & 0.00163 & 0.00327 & 0.00156 \\
\hline N5846_24 & $0.98(2)$ & $0.93(1)$ & $0.90(2)$ & $21.72(9)$ & $8.0(5)$ & $7.7(4)$ & $7.7(5)$ & 0.00107 & 0.00118 & 0.0026 \\
\hline N5846_25 & $1.09(1)$ & $1.02(1)$ & $0.97(1)$ & $21.18(5)$ & $6.5(3)$ & $6.3(3)$ & $6.4(2)$ & 0.00508 & 0.00374 & 0.0028 \\
\hline N5846_27 & $0.91(3)$ & $0.81(2)$ & $0.84(4)$ & $22.4(2)$ & $6.9(7)$ & $6.0(5)$ & $6.3(9)$ & 0.00099 & 0.00075 & 0.0024 \\
\hline N5846_28 & $0.80(1)$ & $0.81(1)$ & $0.89(2)$ & $21.05(9)$ & $6.5(4)$ & $6.0(3)$ & $5.8(4)$ & 0.00345 & 0.00289 & 0.00406 \\
\hline N5846_31 & $1.02(6)$ & $1.10(5)$ & $1.07(8)$ & $22.2(4)$ & $8(2)$ & $9(2)$ & $8(2)$ & 0.00173 & 0.0018 & 0.00227 \\
\hline N5846_32 & $0.99(2)$ & $0.99(1)$ & $1.05(2)$ & $21.83(9)$ & $10.9(9)$ & $11.3(7)$ & $11.9(9)$ & 0.0016 & 0.00131 & 0.00168 \\
\hline N5846_33 & $0.78(2)$ & $0.79(2)$ & $0.83(2)$ & $23.3(1)$ & 11(1) & 12(1) & $13(1)$ & 0.00362 & 0.00173 & 0.00329 \\
\hline N5846_34 & $1.20(2)$ & $1.23(3)$ & $1.13(2)$ & $22.5(1)$ & $9.5(8)$ & $10(1)$ & $9.4(9)$ & 0.00161 & 0.00255 & 0.00225 \\
\hline N5846_35 & $1.28(4)$ & $1.26(3)$ & $1.20(2)$ & $22.2(1)$ & $7(1)$ & 7.1(9) & $7.3(7)$ & 0.00541 & 0.00358 & 0.00206 \\
\hline N5846_37 & $1.3(1)$ & $1.1(1)$ & $1.2(1)$ & $22.9(6)$ & $11(6)$ & $7(2)$ & $10(6)$ & 0.00111 & 0.00376 & 0.00245 \\
\hline N5846_38 & $1.08(5)$ & $1.02(4)$ & $1.21(5)$ & $21.8(2)$ & $4.0(6)$ & $3.8(5)$ & $4.4(7)$ & 0.00575 & 0.00515 & 0.00399 \\
\hline N5846_39 & $0.74(6)$ & $0.99(3)$ & $1.19(9)$ & 22.1(4) & $6(1)$ & $6.1(6)$ & $7(2)$ & 0.0024 & 0.0019 & 0.00173 \\
\hline N5846_40 & $1.15(7)$ & $1.38(5)$ & $1.18(6)$ & $22.5(3)$ & $10(3)$ & $13(3)$ & $9(2)$ & 0.00661 & 0.0016 & 0.00416 \\
\hline N5846_43 & $1.3(2)$ & $1.8(4)$ & $1.2(2)$ & $23(1)$ & $6(5)$ & - & $4(3)$ & 0.00621 & 0.00222 & 0.00715 \\
\hline N5846_46 & $1.36(4)$ & $1.36(5)$ & $1.45(5)$ & $19.6(2)$ & $3.4(6)$ & $3.3(7)$ & $3.3(8)$ & 0.0148 & 0.0148 & 0.00764 \\
\hline N5846_47 & $0.87(2)$ & $0.82(2)$ & $0.85(2)$ & $22.3(1)$ & $11(1)$ & $10.6(9)$ & $11(1)$ & 0.00337 & 0.00217 & 0.00371 \\
\hline N5846_50 & $1.17(4)$ & $1.27(3)$ & $1.35(4)$ & 21.6(2) & $5.5(8)$ & $5.8(8)$ & $6(1)$ & 0.00782 & 0.00516 & 0.00784 \\
\hline N5846_51 & $1.05(2)$ & $1.21(2)$ & $1.19(2)$ & $20.9(1)$ & $4.9(3)$ & $5.5(5)$ & $5.4(4)$ & 0.00177 & 0.0034 & 0.00283 \\
\hline N5846_52 & $1.14(4)$ & $1.78(9)$ & $1.41(5)$ & $21.7(3)$ & $8(1)$ & $14(8)$ & $10(3)$ & 0.00397 & 0.00245 & 0.00322 \\
\hline N5846_56 & $1.10(2)$ & $1.09(2)$ & $1.10(2)$ & $22.12(9)$ & $15(1)$ & $15(1)$ & $15(1)$ & 0.00268 & 0.00264 & 0.00186 \\
\hline
\end{tabular}

Notes. Surface brightnesses are shown in magnitudes $\operatorname{arcsec}^{-2}$, effective radii in arcseconds. Numbers in parantheses indicate errors of the last significant digit. The quality of the fits is shown in the last three columns.

however confirmed its group membership. Assuming the group distance to be $26.1 \mathrm{Mpc}$, the galaxy has a projected diameter of $D_{25} \sim 7 \mathrm{kpc}$, which implies that the galaxy size is inbetween those of bright and dwarf galaxies. The spectrum of N5846_07 uncovers strong $\mathrm{H} \alpha$ emission and weaker $\mathrm{H} \beta$, [N II], and [S II] features, which are indicative of ongoing star formation. Two spiral arms are identified in the optical image, which could also be interpreted as signatures of ongoing interaction with the nearby irregular dwarf N5846_08, only $21 \mathrm{kpc}$ away.

N5846_41/42 are two large HII regions of the irregular dwarf KKR15 (Huchtmeier et al. 2000; Karachentseva et al. 1999), which are classified as individual galaxies in SDSS. The host galaxy shows an extremely blue colour, the majority of its light originating in the two HII regions. The brighter component, N5846_41, is the bluest object of our sample. Because of the high concentration of its light, N5846_41/42 has by far the highest $S / N$ ratio of all spectra in our sample. Besides the usual $\mathrm{H} \alpha, \mathrm{H} \beta,[\mathrm{O} \mathrm{III}],[\mathrm{N} \mathrm{II}]$, and [S II] features, both spectra also exhibit the weaker Balmer lines $\mathrm{H} \gamma, \mathrm{H} \delta$, and $\mathrm{H} \xi$ along

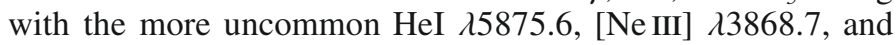
Ar III $\lambda 7135.8$ transitions. Of all emission-line galaxies in our sample, N5846_41/42 has the lowest oxygen abundance with an average value of $12+\log (\mathrm{O} / \mathrm{H})=7.68$ (see Sect. 3.4).
N5846_54 and N5846_55 were both classified as S0 galaxies in the MTT05 sample and are the only objects of our work that have photometric substructures. With projected linear sizes of $D_{25} \sim 4$ and $7 \mathrm{kpc}$, respectively, these objects have, as for N5846_07, sizes that are in-between those of dwarf and bright galaxies. The surface brightness profile of N5846_55 exhibits a rather flat gradient out to 14 arcsec, wherefrom the light distribution falls far more steeply, indicative of a possible bar feature (Gadotti et al. 2007). Spectroscopically, both galaxies exhibit ongoing star formation.

\subsection{Spatial distribution of morphological types}

We studied the morphology distribution for the low-luminosity galaxy population in the NGC 5846 system for both the earlyand late-type populations. Figure 7 illustrates the clustering of both the $\mathrm{dE}$ and dIrr samples with the projected number density shown separately for each morphological type. The diagram displays the same field of view presented in Fig. 1. Density contours were created using a grid with a spatial resolution of $0.5^{\circ} \times 0.5^{\circ}$. The segregation between early- and late-type lowluminosity galaxies in the NGC 5846 system is clearly evident: dwarf ellipticals are found predominantly in the vicinity 


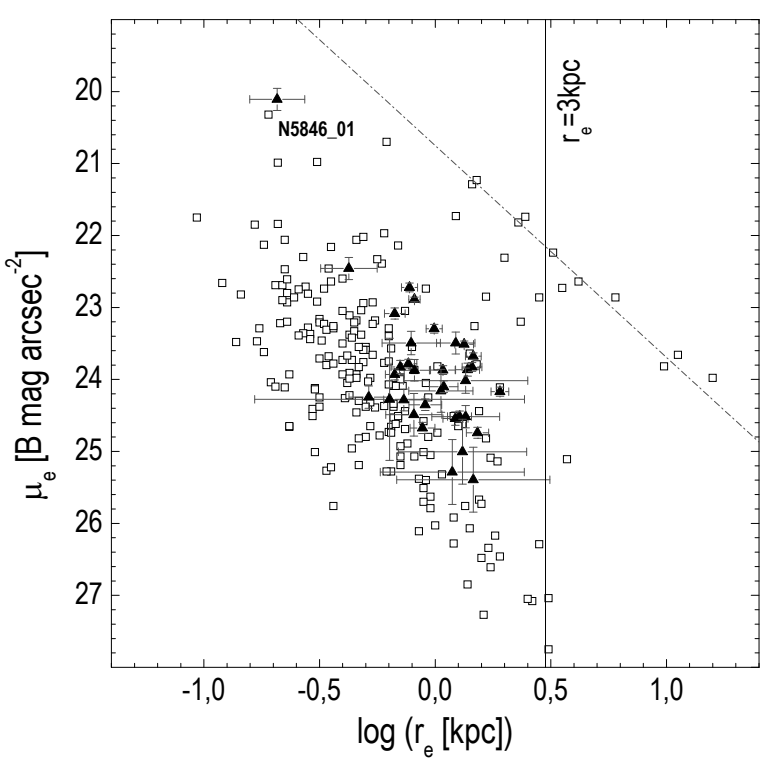

Fig. 5. $\mu_{\mathrm{e}}-\log \left(r_{\mathrm{e}}\right)$ plane for early-type galaxies. Effective surface brightnesses are shown in the Johnson $B$ band. The vertical solid line separates bright and regular ellipticals, while the dash-dotted line represents the Hamabe-Kormendy relation for elliptical galaxies and bulges. Black triangles indicate the NGC 5846 faint galaxy sample. Open squares refer to the groups studied by Khosroshahi et al. (2004) for comparison.

of bright galaxies, while irregular systems are clearly detached from this distribution and diffusely arranged in the outer regions of the group. Morphology shows also a strong correlation with the projected number density. Early-type dwarfs populate the high-density regions around NGC 5846 and NGC 5813, while irregulars show comparatively low agglomeration. Moreover, the satellite morphologies resemble the morphology of their elliptical hosts (NGC 5846 and NGC 5813). Similar results were found by Weinmann et al. (2006), who, on the basis of a large sample of SDSS groups, inferred that the satellite morphologies in groups correlate strongly with the morphology of the central host galaxy. In terms of the radial morphology distributions, Fig. 7 indicates that the projected number density of the early-type population exhibits a much steeper radial gradient than the irregular subsample. In addition to the optical picture, the diffuse X-ray component also correlates spatially with the early-type dwarf distribution (see MTT05); this component should represent the dark matter potentials of the group. We also studied the distribution of nucleated dwarfs within the NGC 5846 system shown in Fig. 8. With the exceptions of two objects, all nucleated dwarfs are found to be in the vicinity $(\sim 260 \mathrm{kpc})$ of NGC 5846 and NGC 5813. Research on the distribution of nucleated dwarfs in the Virgo cluster has also shown that these nuclei are predominantly found in the central regions of the cluster and not in the cluster outskirts (Binggeli \& Cameron 1991). Oh \& Lin (2000) argued that this could be explained by assuming the nuclei to be the result of the orbital decay of globular clusters. By means of a series of numerical simulations, they showed that dwarf galaxies exposed to a little external tidal perturbation dynamical friction can reproduce the significant orbital decays of globular clusters and the formation of compact nuclei within a Hubble time. Thus, a larger fraction of nucleated dwarfs is expected in the centres of galaxy clusters, where the extragalactic tidal perturbation tends to preserve the integrity of dwarf galaxies, unlike in the outskirts, where this perturbation tends to be disruptive.

\subsection{Emission-line dwarfs}

Fluxes measured from $\mathrm{H} \alpha, \mathrm{H} \beta,\left[\mathrm{N}_{\mathrm{II}}\right] \lambda \lambda 6548.1,6583.4 \AA$, [O III] $\lambda \lambda 4958.9,5006.8 \AA$, and [S II] $\lambda \lambda 6717.0,6731.3 \AA$ spectral lines and star formation rates and oxygen abundances were measured for dwarfs showing emission lines in their spectra. The data are presented in Table 3. Star formation rates (SFRs) were derived by means of $\mathrm{H} \alpha$ fluxes using the Kennicutt (1998) relation: $\operatorname{SFR}\left(M_{\odot}\right.$ year $\left.^{-1}\right)=7.9 \times 10^{-42} L(\mathrm{H} \alpha)\left(\mathrm{erg} \mathrm{s}^{-1}\right)$. H $\alpha$ extinction was taken into account using an average extinction value of $A(\mathrm{H} \alpha)=0.95$ mag based on Kennicutt (1983) and Niklas et al. (1997). In addition, Galactic extinction $A_{\mathrm{R}}=0.15 \mathrm{mag}$ was also taken into account so that a total extinction value of $1.10 \mathrm{mag}$ for $\mathrm{H} \alpha$ was allowed. Errors in the star formation rates were estimated by assuming $\mathrm{H} \alpha$ photon noise to be the dominant error source. Star formation rates of galaxies exhibiting $\mathrm{H} \alpha$ fluxes with $S / N<15$ are not listed in Table 3. Emission characteristics were analysed by calculating the line flux ratios [N II] $\lambda 6583 / \mathrm{H} \alpha,[\mathrm{O} \mathrm{III}] \lambda 5007 / \mathrm{H} \beta$, and [S II] $(\lambda 6716+\lambda 6731) / \mathrm{H} \alpha$ according to Veilleux \& Osterbrock (1987). Oxygen abundances were estimated using the $N_{2}$ index ([N II] $\lambda 6583 / \mathrm{H} \alpha$ ) method as defined by Denicoló et al. (2002): $12+\log (\mathrm{O} / \mathrm{H})=9.12( \pm 0.05)+0.73( \pm 0.10) \times N_{2}$. Extinction and reddening effects and particular absorption components related to $\mathrm{H} \alpha$ and $\mathrm{H} \beta$ can considerably affect line flux measurements. A correction was applied to the emission-line galaxies by taking $\mathrm{H} \alpha$ extinction into account and assuming a Balmer decrement of $\mathrm{H} \alpha / \mathrm{H} \beta=2.85$ for $\mathrm{HII}$ region-like galaxies (Veilleux \& Osterbrock 1987) to consider $\mathrm{H} \beta$ extincion too.

Figure 9 distinguishes HII region-like galaxies from AGNs, such as Seyferts or LINERS, according to the diagnosis proposed by Veilleux \& Osterbrock (1987). All emission-line galaxies can be explained by photoionization. Only a few bright galaxies have SDSS spectra. Furthermore, their $\mathrm{H} \beta$ flux is strongly contaminated by the old stellar population, which makes a proper measurement of the line ratio problematic. In addition, the [O III] feature at $\lambda 5007 \AA$ is not seen in most of the investigated bright galaxies. However, on the basis of the measurements of [N II] $\lambda 6583 / \mathrm{H} \alpha$ and [S II] $(\lambda 6716+\lambda 6731) / \mathrm{H} \alpha$ ratios alone, all these objects can be classified as HII region-like objects. There is no evident correlation between star formation activity and the location of emission-line dwarfs within the group. Lequeux et al. (1979) suggested that the oxygen abundances correlate with total galaxy mass for irregular galaxies with more massive galaxies exhibiting higher metal content. Since the galaxy mass is a poorly known parameter, the metallicityluminosity relation instead of the mass-metallicity relation is usually considered. Figure 10 shows the oxygen abundance of emission-line dwarfs measured by means of the $N_{2}$ method versus absolute blue magnitude. Most of our dwarfs lie above the metallicity-luminosity relation of Richer \& McCall (1995). Only the two HII regions N5846_41/42 of KKR15 (Huchtmeier et al. 2000) (see Sect. 3.2) and N5846_12 fall below this sequence. The error bars merely reflect the uncertainty in the $N_{2}$ flux ratio and the error in the linear least squares fit of Denicoló et al. (2002).

\section{Discussion and conclusions}

We have undertaken an analysis of the photometric and spectroscopic properties of the low-luminosity galaxy population of the NGC 5846 group of galaxies located in the Virgo III Cloud of galaxies in the Local Supercluster. This group is of particular interest because it is the most massive of only three dense groups 

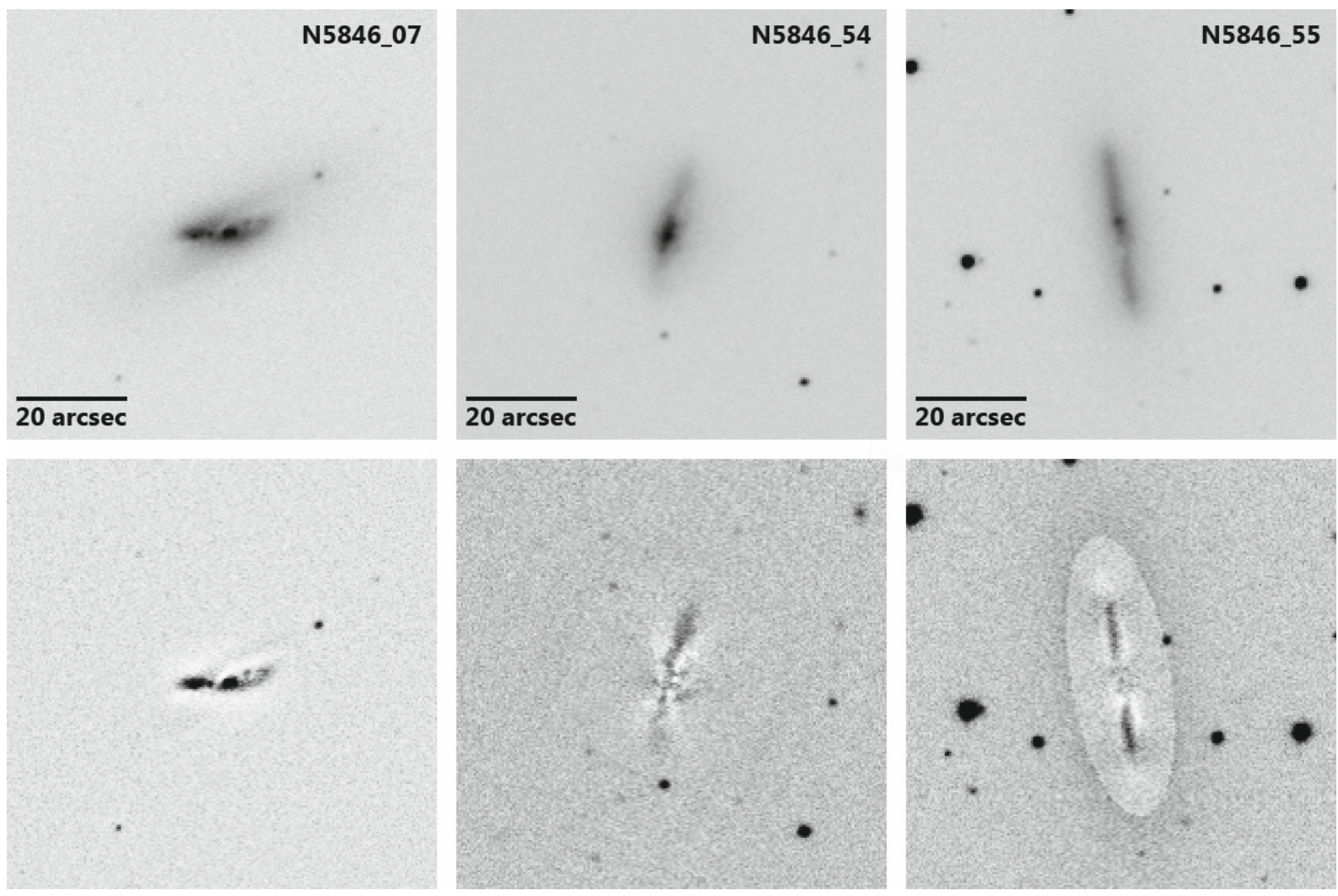

Fig. 6. Modeling of faint galaxies with significant fine structure. Figures show SDSS $r^{\prime}$ band images (upper panel) and residual images (lower panel) of N5846_07, N5846_54 and N5846_55. Since no Sérsic model was obtained for N5846_07, Gaussian smoothing was applied to the galaxy.

(NGC 4274, NGC 5846, and M96) in the Local Supercluster that are dominated by elliptical and lenticular galaxies, and is the third most massive aggregate of early-type galaxies (after the Virgo and Fornax clusters) in the local universe. Of the 19 bright galaxies in our sample, E and S0 galaxies constitute $\sim 58 \%$. Seven new group members in the outer regions $\left(>1.33^{\circ}\right)$ of the group have been identified using SDSS and NED redshifts, complementing existing member catalogues.

The dwarf-to-giant-ratio (DGR) of the NGC 5846 system is high: our sample of spectroscopically determined group members yields a DGR of $\sim 3$. Taking into account the photometric classification of group members to $M_{\mathrm{R}}=-10$ by MTT05 this value could rise to $\sim 12$. Ferguson \& Sandage (1991) demonstrated that the DGR increases with the richness of a group and corresponds to an early-type-dwarf-to-giant-ratio (EDGR) (i.e., $\mathrm{dE}+\mathrm{dE}, \mathrm{N}+\mathrm{dS} 0$ to $\mathrm{E}+\mathrm{S} 0$ ) of 5.77 for the Virgo cluster and 3.83 for the Fornax cluster to $M_{\mathrm{B}}=-13.5$. For the Coma cluster, Secker \& Harris (1996) state an EDGR of $5.80 \pm 1.33$ to $M_{\mathrm{B}}=-13.5$. Our data yield an EDGR of 2.69 for the NGC 5846 system to our faintest dwarf $\left(M_{\mathrm{B}}=-13.32\right)$. This value shows that the NGC 5846 system has fewer early-type dwarfs per giant early-type galaxy than clusters but still a much larger EDGR than other groups (Leo: 1.48, Dorado: 1.44, NGC 1400: 2.08; all down to $M_{\mathrm{B}}=-13.5$, see Ferguson \& Sandage 1991), indicating that NGC 5846 is indeed one of the more massive aggregates in the Local Supercluster.

A colour-magnitude diagram of the investigated group members contains a red sequence displaying the well-known trend of early-type galaxies exhibiting redder colours (higher metallicities) at higher luminosities (Caldwell 1983). In contrast, irregular dwarfs do not form a sequence but show a large spread in colours. There is no evident segregation between bright galaxies and dwarfs.

Photometric scaling relations have been studied for earlytype dwarfs and compared with the relations derived by BJ98 for Virgo cluster dwarf ellipticals. If bright ellipticals and earlytype dwarfs share a similar evolution, a continous sequence in scaling relations with respect to the galaxy luminosity would be expected. It is known that in the surface-brightness-luminosity $(\mu-M)$ plane (Kormendy 1985; Kormendy et al. 2009), earlytype dwarfs and bright ellipticals follow different trends, implying that dwarf galaxies are a distinct class of objects that have followed different evolutionary paths to ordinary ellipticals. Our data match the trends of Virgo cluster dEs suggesting a similar structure and origin of the dwarfs in both the Virgo cluster and the NGC 5846 group. However, one special object falling off the main cloud of early-type dwarfs is N5846_01. It is the closest dwarf to NGC 5846 and shows a comparatively high surface brightness and compactness compared to the other dwarfs investigated. In the $\mu_{0_{B}}-M_{\mathrm{B}}$ plane, the object lies outside the $2 \sigma$ limit compared to the relation of BJ98. Owing to its proximity to NGC 5846, the galaxy has probably been affected by tidal stripping and can therefore be classified as an ultra compact dwarf. This idea is also supported by the nearby object NGC 5846A, the elliptical companion to NGC 5846, having a similar morphological appearance, though being too bright to be considered as a dwarf. Indeed, NGC 5846A is one of the extremely rare compact ellipticals like M32, the prototype of this class. N5846_01 confirms the idea that the structural differences of compact ellipticals and ordinary dEs can be explained if the compacts formed within the potential well of another massive galaxy, whereas dEs evolved as isolated systems (Burkert 1994). Our data also show that early-type dwarfs with high ellipticity tend to have 


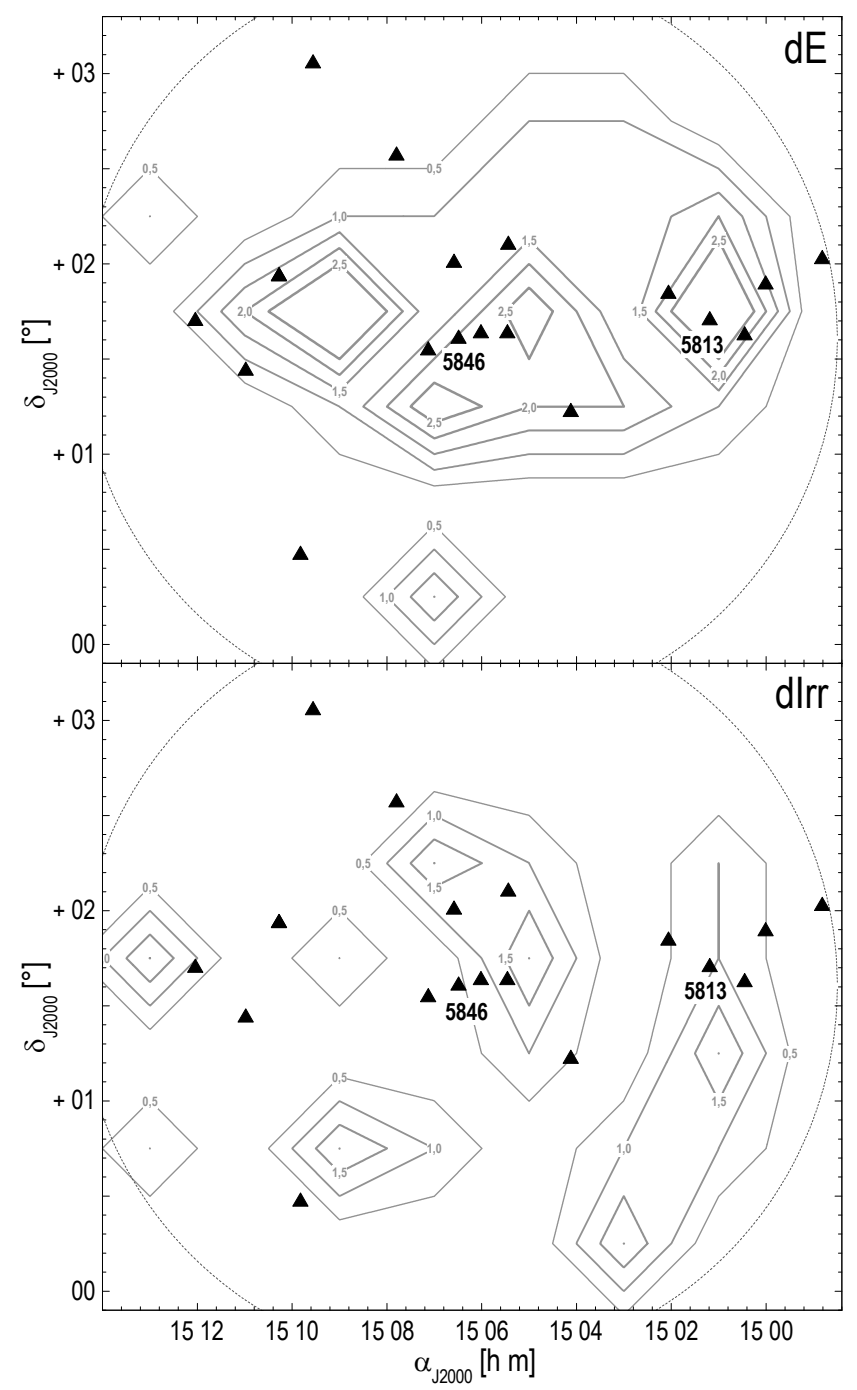

Fig. 7. Clustering properties of both early and late-type dwarfs of the NGC 5846 system. Contours are linearly spaced and show the projected number density of the distinct morphological populations. Triangles refer to bright group members. The values indicate the number of galaxies per 0.25 square degree.

shallower surface brightness profiles. We also checked the location of the NGC 5846 low-luminosity galaxy population in the $\mu_{\mathrm{e}}-\log \left(r_{\mathrm{e}}\right)$ plane with respect to the Hamabe-Kormendy relation, which is a photometric projection of the fundamental plane of galaxies relating the logarithm of effective radii and effective surface brightnesses linearly. The NGC 5846 dwarfs are regular galaxies according to the classification of Capaccioli et al. (1992), indicating that these systems are the building blocks of more massive galaxies, in agreement with $\Lambda$ CDM cosmology. Moreover, the dwarfs are comparable to the dwarfs from $\mathrm{X}$-ray dim and X-ray bright groups studied by Khosroshahi et al. (2004).

Scaling relations for dwarf ellipticals are not the only means of studying their origin and evolution. The study of morphological segregation within clusters and groups also provides the opportunity to test the formation and environmental dependence of the faint galaxy population. The well-known morphologydensity relation (Dressler 1980) is not only restricted to the normal Hubble types but is also found to apply to dwarf galaxies (Binggeli et al. 1987). This relation has been investigated in detail in our immediate vicinity, the Local Group (Grebel 1999).

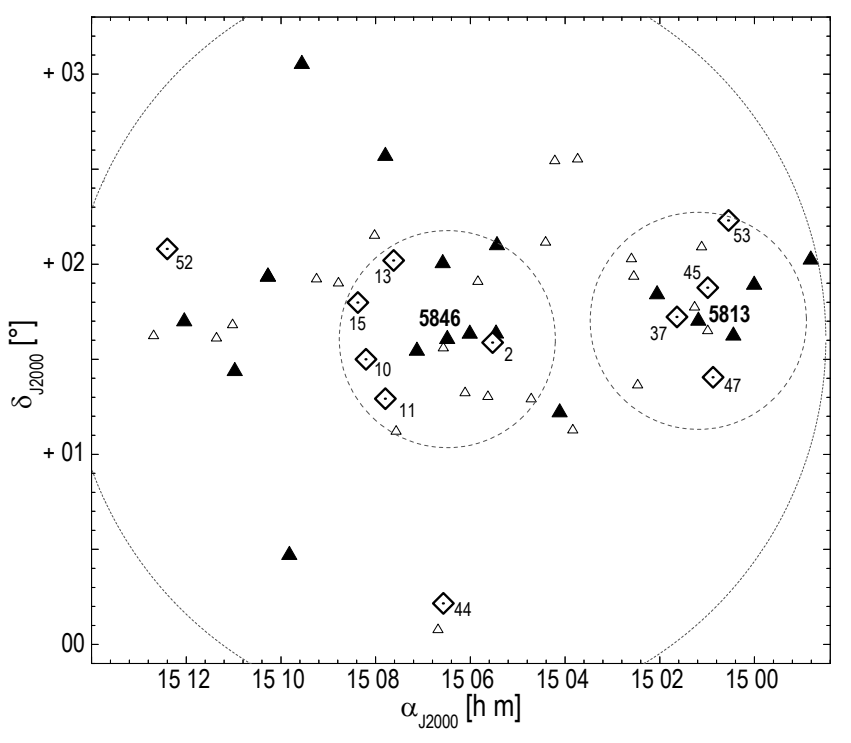

Fig. 8. Distribution of nucleated dwarfs in the NGC 5846 group. Black triangles are bright group members, open triangles dEs without nucleus. Diamonds show nucleated dwarfs, concentrated around NGC 5846 and NGC 5813 (circles indicate a radius of $260 \mathrm{kpc}$ ).

We confirm this morphology-density relation for dwarf galaxies in the NGC 5846 system. While early-type dwarfs are concentrated around the two massive ellipticals NGC 5846 and NGC 5813, objects of irregular type are more randomly distributed and found predominantly in the outer regions. This morphological segregation could be explained by gas stripping due to the infall of the dwarfs towards the group centre. This scenario was also investigated by Weinmann et al. (2006) and Park et al. (2008), who inferred that satellite morphologies in groups tend to be similar to those of their hosts. Because of the comparatively high density of the NGC 5846 system and the hot and dense halo gas present around NGC 5846 and NGC 5813, the dependence of the satellite morphology on host morphology can be interpreted by the hydrodynamic and radiative interaction of the hot X-ray gas of the two host galaxies with their surrounding satellites. We have also checked the distribution of nucleated dwarfs in the NGC 5846 system with respect to non-nucleated ones. Interestingly, nearly all dwarfs that contain a nucleus superimposed on their underlying smooth surface brightness profile are found in the vicinity of NGC 5846 and NGC 5813 within a radius of $\sim 260 \mathrm{kpc}$. This is in agreement with the work of Binggeli \& Cameron (1991) for the Virgo cluster, who show that dwarfs located near the centre of the cluster are mostly nucleated while those in the outskirts are non-nucleated. Assuming that the formation of the nuclei is related to the orbital decay of globular clusters, Oh \& Lin (2000) argued that this could be explained by extragalactic tidal perturbation, which tends to preserve the integrity of dwarf galaxies at cluster centres but tends to disrupt dwarf galaxies in their outskirts.

Only two objects of the low-luminosity galaxy population exhibit photometric fine structure. These galaxies exhibit ongoing star formation and diameter sizes that are in-between those of dwarf and bright galaxies. This lack of photometric peculiarities in the low-luminosity galaxy population emphasises that the NGC 5846 system is an old, well-evolved aggregate, where no recent interactions between individual members have occurred. Emission characteristics and star formation rates of the irregular dwarfs exhibit typical values for low-luminosity galaxies, additionally supporting the idea of a system that does not exhibit 
Table 3. Spectral properties of emission-line dwarfs.

\begin{tabular}{|c|c|c|c|c|c|c|c|c|c|c|}
\hline Galaxy & $\begin{array}{c}\mathrm{H} \beta \\
\lambda 4861.3\end{array}$ & $\begin{array}{c}{[\mathrm{O} \text { III }]} \\
\lambda 4958.9\end{array}$ & $\begin{array}{c}{[\mathrm{O} \text { III] }} \\
\lambda 5006.8\end{array}$ & $\begin{array}{c}{[\mathrm{N} \text { II }]} \\
\lambda 6548.1\end{array}$ & $\begin{array}{c}\mathrm{H} \alpha \\
\lambda 6562.8\end{array}$ & $\begin{array}{c}{[\mathrm{N} \text { II }]} \\
\lambda 6583.4\end{array}$ & $\begin{array}{c}{[\mathrm{S} \text { II] }} \\
\lambda 6717.0\end{array}$ & $\begin{array}{c}{[\mathrm{S} \text { II] }} \\
\lambda 6731.3\end{array}$ & $\begin{array}{c}\text { SFR } \\
M_{\odot} \mathrm{yr}^{-1}\end{array}$ & $\begin{array}{c}12+\log \mathrm{O} / \mathrm{H} \\
{[\operatorname{dex}]}\end{array}$ \\
\hline N5846_06 & 348.1 & 45.8 & 128.4 & 14.0 & 992.0 & 51.7 & 66.6 & 47.7 & $0.0064(3)$ & $8.2(1)$ \\
\hline N5846_07 & 619.6 & - & 4.2 & 62.5 & 1765.9 & 173.7 & 107.2 & 81.3 & $0.0114(4)$ & $8.4(1)$ \\
\hline N5846_08 & - & - & - & 7.7 & 51.0 & 7.2 & 12.5 & 8.0 & - & $8.5(1)$ \\
\hline N5846_09 & 79.0 & - & 18.9 & 3.1 & 225.1 & 8.5 & 21.4 & 16.5 & - & $8.1(1)$ \\
\hline N5846_12 & 300.1 & 93.9 & 270.4 & 7.9 & 855.4 & 16.6 & 47.5 & 26.7 & $0.0055(3)$ & $7.8(2)$ \\
\hline N5846_14 & 409.9 & 71.9 & 212.0 & 10.1 & 1168.3 & 47.8 & 89.3 & 64.0 & $0.0075(4)$ & $8.1(2)$ \\
\hline N5846_15 & - & - & - & - & 24.2 & - & - & - & - & - \\
\hline N5846_17 & - & - & - & - & 46.7 & 9.1 & 6.5 & 6.8 & - & $8.6(1)$ \\
\hline N5846_18 & - & - & - & - & 30.7 & - & 7.7 & 7.4 & - & - \\
\hline N5846_19 & 43.7 & - & 19.3 & 8.6 & 124.6 & 14.0 & 21.3 & 12.3 & - & $8.4(1)$ \\
\hline N5846_20 & 171.1 & 5.4 & 39.9 & 17.5 & 487.5 & 42.4 & 51.4 & 37.5 & - & $8.4(1)$ \\
\hline N5846_27 & - & - & - & - & 38.8 & - & 7.9 & - & - & - \\
\hline N5846_28 & 298.8 & 15.6 & 50.1 & 21.2 & 851.5 & 67.1 & 64.5 & 52.6 & $0.0055(3)$ & $8.3(1)$ \\
\hline N5846_33 & - & - & - & - & 29.7 & - & - & - & - & - \\
\hline N5846_38 & 103.8 & 12.1 & 40.8 & 8.6 & 295.8 & 20.5 & 16.2 & 14.4 & - & $8.3(1)$ \\
\hline N5846_39 & 34.0 & - & 10.4 & - & 96.8 & 9.3 & 19.2 & 12.7 & - & $8.4(1)$ \\
\hline N5846_40 & - & - & - & - & 42.8 & - & 9.3 & 5.9 & - & - \\
\hline N5846_41 & 2576.2 & 1222.0 & 3664.0 & 20.6 & 7342.2 & 68.1 & 182.0 & 127.8 & $0.0473(9)$ & $7.6(2)$ \\
\hline N5846_42 & 914.9 & 475.5 & 1385.0 & 10.9 & 2607.5 & 31.4 & 76.4 & 48.9 & $0.0168(5)$ & $7.7(2)$ \\
\hline N5846_46 & 223.7 & 15.3 & 43.8 & 16.6 & 637.6 & 51.7 & 53.9 & 43.1 & $0.0041(3)$ & $8.3(1)$ \\
\hline N5846_49 & 28.9 & - & 8.7 & - & 82.3 & 2.4 & 5.3 & 7.7 & - & $8.0(1)$ \\
\hline N5846_50 & - & - & - & - & 32.2 & - & - & - & - & - \\
\hline N5846_51 & 62.6 & 8.9 & 38.8 & 8.6 & 178.4 & 15.8 & 24.5 & 20.9 & - & $8.4(1)$ \\
\hline N5846_54 & 290.9 & - & 45.4 & 31.4 & 829.2 & 89.8 & 75.4 & 57.6 & $0.0053(3)$ & $8.4(1)$ \\
\hline N5846_55 & 461.1 & - & 17.9 & 47.0 & 1314.2 & 140.1 & 81.2 & 61.9 & $0.0085(4)$ & $8.4(1)$ \\
\hline N5846_56 & 40.6 & - & 15.6 & 12.0 & 115.7 & 10.5 & 14.1 & 10.2 & - & $8.3(1)$ \\
\hline
\end{tabular}

Notes. Fluxes present the integrals of the fitted Gaussians and are given in units of $10^{-17} \mathrm{erg} \mathrm{s}^{-1} \mathrm{~cm}^{-2}$. $\mathrm{H} \alpha$ and $\mathrm{H} \beta$ fluxes have been corrected for extinction. Numbers in parentheses indicate errors of the last significant digit.

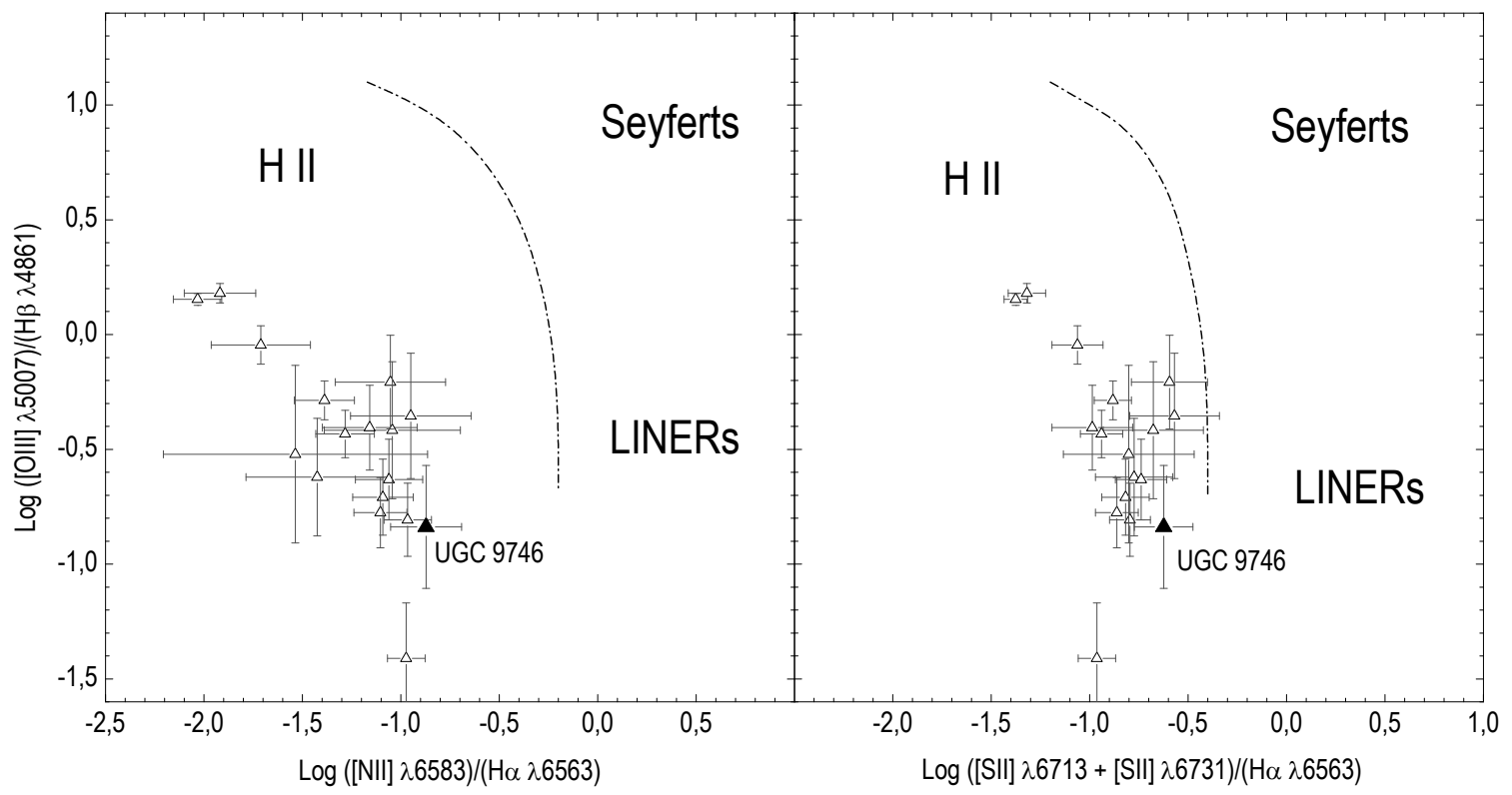

Fig. 9. Classification of emission-line galaxies in the NGC 5846 group according to Veilleux \& Osterbrock (1987). The line separates HII regionlike galaxies from AGNs. All measured objects show flux ratios explained by simple photoionization.

recent activity that is enhancing star formation. Oxygen abundances have been derived indirectly via the $N_{2}$ index method proposed by Denicoló et al. (2002). Our abundances show a large scatter $(\sigma=0.36 \mathrm{dex})$ with respect to the metallicity-luminosity relation of Richer \& McCall (1995) due to the intrinsic scatter in the $N_{2}$ method itself. Nevertheless, all objects outside $1 \sigma$ confidence intervals lie above the metallicity-luminosity relation, indicating a comparatively high oxygen abundance for irregular dwarfs in the NGC 5846 group.

Our study of the low-luminosity galaxy population of the NGC 5846 group has found that the dwarf galaxies in this massive group match the trends observed for dwarf galaxies in other aggregates, which implies that they have similar formation and evolution scenarios. The spatial distribution of morphological 


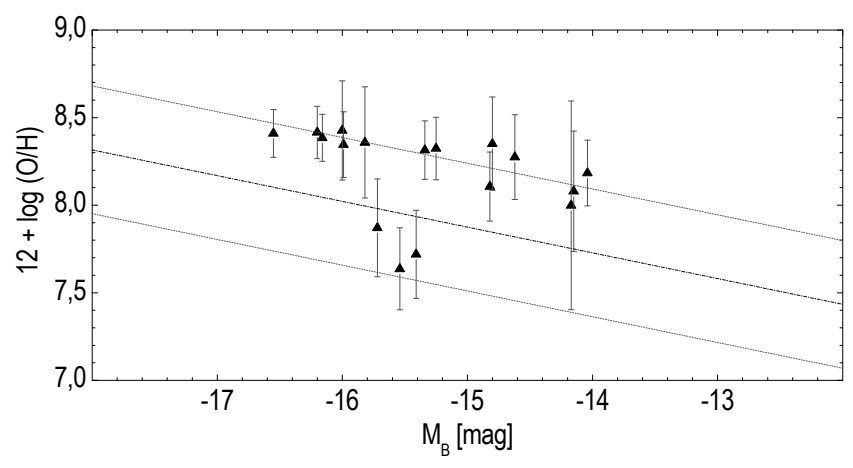

Fig. 10. Oxygen abundances versus abolute B magnitudes of NGC 5846 irrgeluar dwarfs. The abundances were determined indirectly using the $N_{2}$ method as proposed by Denicoló et al. (2002). The dash-dotted line shows the metallicity-luminosity relation of Richer \& McCall (1995). One- $\sigma$ deviations are shown as dashed lines.

types suggests that the structural properties of dwarf galaxies are clearly dependent on the location within the group. The structural properties of the dwarfs confirm the evolved state of the system. The group remains unusual because of predominant early-type morphology of its members, the strong X-ray emission, and the high dwarf-to-giant-ratio compared to other groups.

Acknowledgements. We acknowledge the useful comments from the anonymous referee which helped to improve the paper. We are also very grateful to the language editor, Claire Halliday, for her careful reading of the manuscript. P.E. has been supported by the University of Vienna in the frame of the Initiativkolleg (IK) The Cosmic Matter Circuit I033-N. This work has made use of the astronomical data reduction software IRAF which is distributed by the National Optical Astronomy Observatories, which are operated by the Association of Universities for Research in Astronomy Inc., under cooperative agreement with the National Science Foundation.

\section{References}

Adelman-McCarthy, J. K., Agüeros, M. A., Allam, Sahar, S., et al. 2006, ApJS, 162,38

Bertschinger, E. 1985, ApJS, 58, 39

Biermann, P. L., Kronberg, P. P., \& Schmutzler, T. 1989, A\&A, 208, 22

Binggeli, B., \& Cameron, L. M. 1991, A\&A, 252, 27

Binggeli, B., \& Jerjen, H. 1998, A\&A, 333, 17 [BJ98]

Binggeli, B., Tammann, G. A., \& Sandage, A. 1987, AJ, 94, 251

Burkert, A. 1994, MNRAS, 266, 877

Caldwell, N. 1983, AJ, 88, 804

Capaccioli, M., Caon, N., D’Onofrio, M., \& Trevisani, S. 1992, MmSAI, 63, 509 Capelato, H. V., de Carvalho, R. R., \& Carlberg, R. G. 1995, ApJ, 451, 525

Carrasco, E. R., Mendes de Oliveira, C., \& Infante, L. 2006, AJ, 132, 1796

Côté, S., Freeman, K. C., Carignan, C., \& Quinn, P. J. 1997, AJ, 114, 1313

Denicoló, G., Terlevich, R., \& Terlevich, E. 2002, MNRAS, 330, 69

de Vaucouleurs, G. 1975, Stars and Stellar Systems, 9, 557

di Serego Alighieri, S., Vernet, J., Cimatti, A., Lanzoni, B., et al. 2005, A\&A, 442,125

Dressler, A. 1980, ApJ, 236, 351

Evstigneeva, E. A., de Carvalho, R. R., Ribeiro, A. L., \& Capelato, H. V. 2004, MNRAS, 349, 1052
Ferguson, H. C. 1989, AJ, 98, 367

Ferguson, H. C., \& Binggeli, B. 1994, A\&ARv, 6, 67

Ferguson, H. C., \& Sandage, A. 1991, AJ, 101, 765

Finoguenov, A., Jones, C., Forman, W., \& David, L. 1999, ApJ, 514, 844

Forbes, D. A., Brodie, J. P., \& Huchra, J. 1997, AJ, 113, 887

Fukugita, M., et al. 1996, AJ, 11, 1748

Gadotti, D. A., Athanassoula, E., Carrasco, L., Bosma, A., et al. 2007, MNRAS, 381,943

Garcia, A. M. 1993, A\&AS, 100, 47

Geller, M. J., \& Huchra, J. P. 1983, ApJS, 52, 61

Gladders, M. D., Lopez-Cruz, O., Yee, H. K. C., \& Kodama, T. 1998, ApJ, 501, 571

Grebel, E. K. 1999, IAUS, 192, 17

Grützbauch, R., Zeilinger, W. W., Rampazzo, R., et al. 2009, A\&A, 502, 473

Hamabe, M., \& Kormendy, J. 1987, IAUS, 127, 379

Heisler, J., Tremaine, S., \& Bahcall, J. N. 1985, ApJ, 298, 8

Higdon, J. L., Buta, R. J., \& Purcell, G. B. 1998, AJ, 115, 80

Hopp, U., Wagner, S. J., \& Richtler, T. 1995, A\&A, 296, 633

Huchtmeier, W. K., Karachentsev, I. D., \& Karachentseva, V. E. 2000, A\&AS, 147,187

Jedrzejewski, R. I. 1987, MNRAS, 226, 747

Jerjen, H., Binggeli, B., \& Freeman, K. C. 2000, AJ, 119, 593

Karachentsev, I. D., Dolphin, A. E., Geisler, D., et al. 2002, A\&A, 383, 125

Karachentseva, V. E., Karachentsev, I. D., \& Richter, G. M. 1999, A\&AS, 135, 221

Kennicutt, R. C. Jr. 1983, ApJ, 272, 54

Kennicutt, R. C. Jr. 1998, ARA\&A, 36, 189

Khosroshahi, H. G., Raychaudhury, S., Ponman, T. J., et al. 2004, MNRAS, 349, 527

Klypin, A., Kravtsov, A. V., Valenzuela, O., \& Prada, F. 1999, ApJ, 522, 82

Kormendy, J. 1985, ApJ, 295, 73

Kormendy, J., Fisher, D. B., Cornell, M. E., \& Bender, R. 2009, ApJS, 182, 216 Lequeux, J., Peimbert, M., Rayo, J. F., Serrano, A., \& Torres-Peimbert, S. 1979, A\&A, 80, 155

Mahdavi, A., Trentham, N., \& Tully, R. B. 2005, AJ, 130, 1502 [MTT05]

Mulchaey, J. S., Davis, D. S., Mushotzky, R. F., \& Burstein, D. 2003, ApJS, 145, 39

Nieto, J.-L., Davoust, E., Bender, R., \& Prugniel, P. 1990, A\&A, 230, 17

Niklas, S., Klein, U., \& Wielebinski, R. 1997, A\&A, 322, 19

Oh, K. S., \& Lin, D. N. C. 2000, ApJ, 543620

Park, C., Gott, J. R. III, \& Choi, Y.-Y. 2008, ApJ, 674, 784

Peterson, R. C., \& Caldwell, N. 1993, AJ, 105, 1411

Prieto, M., Gottesman, S. T., Aguerri, J. L., \& Varela, A. 1997, AJ, 114, 1413

Richer, M. G., \& McCall, M. L. 1995, ApJ, 445, 642

Rothberg, B., \& Joseph, R. D. 2004, AJ, 128, 2098

Sandage, A. 1986, ApJ, 307, 1

Sandage, A., \& Binggeli, B. 1984, AJ, 89, 919

Secker, J., \& Harris, W. E. 1996, ApJ, 469, 623

Sérsic, J. L. 1968, Atlas de galaxias australes, Argentina: Observatorio Astronomico

Schlegel, D. J., Finkbeiner, D. P., \& Davis, M. 1998, ApJ, 500, 525

Smith, J. A., Tucker, D. L., Kent, S., et al. 2002, AJ, 123, 2121

Temporin, S., Weinberger, R., Galaz, G., \& Kerber, F. 2003, ApJ, 587, 660

Trinchieri, G., \& Goudfrooij, P. 2002, A\&A, 386, 472

Tully, R. B. 1982, ApJ, 257, 389

Tully, R. B. 1988, ApJ, 96, 73

Tully, R. B., \& Fisher, J. R. 1988, Nearby Galaxies Catalog (Cambridge University Press)

Veilleux, S., \& Osterbrock, D. E. 1987, ApJS, 63, 295

Weinmann, S. M., van den Bosch, F. C., Yang, X., \& Mo, H. J. 2006, MNRAS, 366, 2

Zabludoff, A. I. 1999, IAUS, 192, 433

Zabludoff, A. I., \& Mulchaey, J. S. 1998, ApJ, 496, 39

Ziegler, B. L., Saglia, R. P., Bender, R., Belloni, P., et al. 1999, A\&A, 346, 13 